\title{
EQUIVARIANT COHOMOLOGY OF RATIONALLY SMOOTH GROUP EMBEDDINGS
}

\author{
RICHARD P. GONZALES
}

\begin{abstract}
We describe the equivariant cohomology ring of rationally smooth projective embeddings of reductive groups. These embeddings are the projectivizations of reductive monoids. Our main result describes their equivariant cohomology in terms of roots, idempotents, and underlying monoid data. Also, we characterize those embeddings whose equivariant cohomology ring is obtained via restriction to the associated toric variety. Such characterization is given in terms of the closed orbits.
\end{abstract}

\section{INTRODUCTION AND MOTIVATION}

Let $G$ be a connected complex reductive algebraic group, $B \subset G$ a Borel subgroup, and $T \subset B$ a maximal torus. A $G$-variety is called spherical if it is normal and contains a dense $B$-orbit. A nice feature of spherical $G$-varieties is that they contain only finitely many $B$-orbits and $G$-orbits. This yields a complete description of the geometry of spherical varieties in terms of certain combinatorial objects of discrete convex geometry. See [T2 and [P1] for an up-to-date discussion of spherical varieties and a comprehensive bibliography. In this paper, we focus on a special yet remarkable class of spherical varieties, namely, group embeddings.

An irreducible algebraic variety is called an embedding of $G$, or a group embedding, if it is a normal $G \times G$-variety containing an open orbit isomorphic to $G$ itself, where $G \times G$ acts on $G$ by left and right multiplication. When $G$ is a torus, we get back the notion of toric varieties. Group embeddings are spherical $G \times G$-varieties due to the Bruhat decomposition. Affine embeddings of $G$ are nothing but reductive monoids having $G$ as group of units $\mathrm{Ri}$. Recall that an algebraic monoid is an algebraic variety equipped with an associative product map, which is a morphism of varieties and admits an identity element. An affine algebraic monoid is called reductive if it is irreducible, normal, and its unit group is a reductive algebraic group. Reductive monoids have been intensively studied in the works of Putcha, Renner, Vinberg, Rittatore, Brion, and others. See $[\mathrm{Pu},[\mathrm{R} 8, \mathrm{Br} 7, \mathrm{~T} 2]$ and the references therein.

Let $M$ be a reductive monoid with zero and unit group $G$. Then there exists a central one-parameter subgroup $\epsilon: \mathbb{G}_{m} \rightarrow G$ such that $\lim _{t \rightarrow 0} \epsilon(t)=0$. Moreover, the quotient space $\mathbb{P}_{\epsilon}(M):=(M \backslash\{0\}) / \epsilon\left(\mathbb{G}_{m}\right)$ is a normal projective embedding of the quotient group $G / \epsilon\left(\mathbb{G}_{m}\right)$. These varieties were introduced by Renner in his study of algebraic monoids [R1, R4, R6]. Projective embeddings of connected reductive groups are exactly the projectivizations of reductive monoids [R1].

Let $X$ be a complex algebraic variety of dimension $n$. Cohomology, in this article, is always considered with rational coefficients. We say that $X$ is rationally smooth if we have $H^{m}(X, X \backslash\{x\})=0$ for $m \neq 2 n$, and $H^{2 n}(X, X \backslash\{x\})=\mathbb{Q}$, for all $x \in X$. This is precisely the requirement that $X$ is a rational cohomology manifold. 
See $\mathrm{Br} 4$ for a modern account of this key notion. Using chiefly methods from the theory of algebraic monoids, Renner investigated those group embeddings that are rationally smooth [R6], R7]. This class is larger than the class of smooth group embeddings.

Now let $X=\mathbb{P}_{\epsilon}(M)$ be a projective group embedding. The purpose of this article is to determine the equivariant cohomology rings $H_{T \times T}^{*}(X)$ and $H_{G \times G}^{*}(X)$ provided $X$ is rationally smooth. Our main results generalize those of Brion [Br3] and Littelmann-Procesi $[\mathrm{LP}$ for regular group embeddings. The main tool in our description is the theory developed by Goresky-Kottwitz-MacPherson GKM. A key result of GKM theory (Theorem 3.1) gives an explicit presentation of the equivariant cohomology of $T$-skeletal varieties (i.e. complete $T$-varieties with only finitely many $T$-fixed points and $T$-stable curves) whose odd cohomology vanishes. If $X$ is rationally smooth, then $X$ has no cohomology in odd degrees, by a previous result of the author [G1, Theorem 7.4]. Hence, to attain the sought-after descriptions of $H_{T \times T}^{*}(X)$ and $H_{G \times G}^{*}(X)$, we proceed in two steps: first we compute the GKM data of $X$, i.e. $T \times T$-fixed points, $T \times T$-stable curves and the corresponding characters of $T \times T$, in terms of the combinatorial data of $M$. We remark that the calculation of such data is independent of whether or not $X$ is rationally smooth. Afterwards, we specialize it to the case when $X$ is rationally smooth via Theorem 3.1 Our findings increase the applicability of $G K M$ theory in the study of singular group embeddings.

The results and methods of this paper open the way to further developments, e.g., if $X=\mathbb{P}_{\epsilon}(M)$ is any group embedding, then the GKM data obtained here describes the $T \times T$-equivariant operational $K$-theory of $X$, i.e. certain ring of piecewise exponential functions acting on the $T \times T$-equivariant $K$-theory of $X$, see G2. In addition, a description of the module structure of the (rational) equivariant Chow groups of rationally smooth group embeddings, in the spirit of [G1, is obtained in G3. Since $X$ is possibly singular, and working with intersection theory is usually more delicate than with cohomology, the description in G3 is not a straightforward generalization of [G1]. Other techniques are needed. These results will appear elsewhere.

Here is an outline of the paper. In the first part (Sections 1 and 2) we work over an algebraically closed field $\mathbb{k}$ of arbitrary characteristic, since the classification of group embeddings outlined above holds in this generality [BK, Chapter 6]. Section 1 gathers some preliminary notions and results from the theory of reductive monoids. In Section 2 we show that any projective group embedding $\mathbb{P}_{\epsilon}(M)$ is $T \times T$-skeletal and assess the corresponding GKM data. See Theorem 2.1, Theorem 2.5] and Subsection 2.4.

In the second part (Sections 3 and 4), we specialize the results of Section 2 to the case when $\mathbb{k}=\mathbb{C}$ and the group embedding $X=\mathbb{P}_{\epsilon}(M)$ is rationally smooth. Subsection 3.1 collects basic facts on GKM theory. Subsection 3.2 provides a short discussion of rationally smooth group embeddings. Subsection 3.3 contains our major results: Theorem 3.8 gives the ultimate description of $H_{T \times T}^{*}(X)$ in terms of the finite combinatorial invariants of $M$, i.e. the roots of $(G, T)$ and the Renner monoid. Theorem 3.10 compares $H_{G \times G}^{*}\left(\mathbb{P}_{\epsilon}(M)\right)$ and $H_{T \times T}^{*}\left(\mathbb{P}_{\epsilon}(\bar{T})\right)$, where $\mathbb{P}_{\epsilon}(\bar{T})$ is the associated torus embedding. We show that, unlike the case of regular embeddings, $H_{G \times G}^{*}\left(\mathbb{P}_{\epsilon}(M)\right)$ is in general a proper subring of $H_{T \times T}^{*}\left(\mathbb{P}_{\epsilon}(\bar{T})\right)^{W}$, where 
$W$ is the Weyl group of $(G, T)$. In Proposition 3.13 we characterize those embeddings for which these two rings coincide; they correspond to the toroidal rationally smooth group embeddings, i.e. those whose closed $G \times G$-orbits are all of the form $G / B \times G / B^{-}$. Section 3 concludes with a description of the non-equivariant cohomology of toroidal rationally smooth group embeddings (Theorem 3.14). Finally, in Section 4, we illustrate the theory with a detailed study of simple projective embeddings, i.e. those with only one closed $G \times G$-orbit (Theorem 4.6).

Acknowledgments. Some of these results were part of the author's doctoral dissertation under the supervision of Lex Renner. I would like to thank him and Michel Brion for their invaluable help. I would also like to thank Kürşat Aker, whose interest in the subject allowed me to present this work at various universities in Turkey, and the Technological Research Council of Turkey (TÜBITAK), for the support that I received, as a postdoctoral fellow, through Projects 107T897 and 109T667. I am also grateful to the German Research Foundation (DFG), Research Grant PE2165/1-1, for its support during the final stages of completing this article. Lastly, I thank the two referees for very helpful comments and suggestions that improved the clarity of the article.

Notation and Conventions. We work over an algebraically closed field $\mathbb{k}$. In Sections 1 and $2, \mathbb{k}$ is of arbitrary characteristic; in Sections 3 and $4, \mathbb{k}=\mathbb{C}$. All algebraic varieties and algebraic groups are assumed to be defined over $\mathbb{k}$. By a variety we mean a separated reduced scheme of finite type over $\mathbb{k}$; in particular varieties need not be irreducible. A point will always mean a closed point.

$G$ denotes a connected reductive linear algebraic group with Borel subgroup $B$ and maximal torus $T \subset B$. The Weyl group of $(G, T)$ is denoted by $W$. Recall that $W=N_{G}(T) / T$, where $N_{G}(T)$ is the normalizer of $T$ in $G$. We denote by $\Xi$ the character group of $T$, and by $\Phi$ (resp. $\Delta$ ) the set of roots (resp. simple roots) of $(G, T)$. So $\Delta \subset \Phi \subset \Xi$. We denote by $s_{\alpha} \in W$ the reflection corresponding to $\alpha \in \Phi$. Observe that $W$ is generated by the simple reflections $\left\{s_{\alpha}\right\}_{\alpha \in \Delta}$. We write $U_{\alpha}$ for the unipotent subgroup of $G$ associated to $\alpha \in \Phi$.

For $w \in W$, denote by $\operatorname{int}(w)$ the inner automorphism of $T$ given by conjugation with (a representative of) $w$. This yields a $W$-action on $\Xi$ via $(w, \chi) \mapsto \chi \circ \operatorname{int}(w)^{-1}$, where $w \in W$, and $\chi \in \Xi$. We denote by $S$ the symmetric algebra over $\mathbb{Q}$ of the abelian group $\Xi$. Let $S^{W} \subset S$ be the subring of $W$-invariants. Then $S$ is a free $S^{W}$-module of rank $|W|$. Furthermore, there is a graded $W$-stable subspace $R \subset S$, isomorphic to the regular representation of $W$, such that $S \simeq R \otimes S^{W}$ as graded $S^{W}$-modules, see e.g. [Hu, Section 3.6].

For a $T$-variety $X$, we denote by $X^{T}$ the fixed point set, and by $i_{T}: X^{T} \rightarrow X$ the inclusion. The (rational) $T$-equivariant cohomology of a complex $T$-variety $X$ is denoted by $H_{T}^{*}(X)$. The $T$-equivariant cohomology of a point identifies to $S$, where each character has degree 2. More generally, $H_{T}^{*}(X)$ is an algebra over $S$.

\section{Preliminaries}

1.1. Reductive monoids. We collect a few crucial results from the theory of reductive monoids. For a complete treatment of the subject, the reader is invitated to consult $[\mathrm{R} 8$ and $[\mathrm{Pu}$. Those interested in a survey of the main ideas may also see [So. The semi-expository article [Br7] contains more recent developments. 
Throughout the paper, $M$ denotes a reductive monoid with zero and unit group $G=G(M)$. There is a natural $G \times G$-action on $M$ given by $(g, h) \cdot a:=g a h^{-1}$. Let $\bar{T} \subset M$ be the Zariski closure of $T$ in $M$. It is known that $\bar{T}$ is normal affine toric variety [ $\mathrm{R} 8$, Theorem 5.4]. Moreover, $\bar{T}=\{x \in M \mid x t=t x$, for all $t \in T\}$, see [R8, proof of Theorem 5.5].

We write $E(M)$ for the idempotent set of $M$, that is, $E(M):=\left\{e \in M \mid e^{2}=e\right\}$. Clearly, $E(M)$ is stable under the conjugation action of $G$. We denote by $E(\bar{T})$ the idempotent set of $\bar{T}$. Observe that $E(\bar{T})$ is invariant under the conjugation action of $W$. Define a partial order on $E(M)$ (and thus on $E(\bar{T}) \subset E(M)$ ) by declaring $f \leq e$ if and only if $f e=f=e f$.

The set $G \backslash M / G$ of $G \times G$-orbits in $M$ is finite (for $M$ is $G \times G$-spherical). Moreover, every $G \times G$-orbit contains an idempotent, since $M=E(M) G$ (see [R8, Theorem 4.2]). The following properties are specially important in the analysis of the $G \times G$-orbit structure of $M$.

- Any idempotent of $M$ is conjugate to one in $\bar{T}[\mathrm{R} 8$, Proposition 3.13].

- If $e, f \in E(M)$, then $G e G=G f G$ if and only if $e$ and $f$ are conjugate under $G$ [R8, Proposition 3.13].

- If $e, f \in E(\bar{T})$ are conjugate under $G$, then they are conjugate under $W$ $\mathrm{Pu}$, Theorem 6.25].

Consequently, there are bijections

$$
G \backslash M / G \longleftrightarrow E(M) / G \longleftrightarrow E(\bar{T}) / W
$$

given by

$$
G e G \longleftrightarrow\left\{g^{-1} g^{-1} \mid g \in G\right\} \longleftrightarrow\left\{w e w^{-1} \mid w \in W\right\}
$$

for $e \in E(\bar{T})$. Here $E(M) / G$ is the set of $G$-conjugacy classes in $E(M)$, and $E(\bar{T}) / W$ is the set of $W$-conjugacy classes in $E(\bar{T})$.

We denote by $\Lambda$ the cross section lattice of $M$ (relative to $T$ and $B$ ). This is the subset of $E(\bar{T})$ defined as

$$
\Lambda:=\{e \in E(\bar{T}) \mid B e=e B e\} .
$$

It turns out that $\Lambda$ can be identified with the (finite) set $G \backslash M / G$ [R8, Theorem 4.5]. Therefore,

$$
M=\bigsqcup_{e \in \Lambda} G e G .
$$

By our previous remarks, we can also identify $\Lambda$ with the set $E(\bar{T}) / W$. In the sequel, we shall use freely these identifications.

Next we define the Renner monoid. Let $R=\overline{N_{G}(T)} \subset M$. If $x \in R$, then $x=w t$ for some $w \in N_{G}(T)$ and $t \in \bar{T}$. Hence, $x T=T x$. In fact, one checks that $R=\{x \in M \mid T x=x T\}$, cf. [R2, p. 309]. It follows that $\mathcal{R}:=R / T=T \backslash R$ has the unique structure of a finite monoid; its group of units is $W$ and its idempotent set is $E(\bar{T})$. Moreover, $\mathcal{R} \simeq E(\bar{T}) \cdot W[\mathrm{R} 8$, Proposition 8.1]. The monoid $\mathcal{R}$ is called the Renner monoid of $M$. Observe that in an expression for $x \in \mathcal{R}$ of the form $x=e w$, with $e \in E(\bar{T})$ and $w \in W$, the idempotent $e$ is uniquely determined, that is, if $x=e w=e^{\prime} w^{\prime}$, with $e, e^{\prime} \in E(\bar{T})$ and $w, w^{\prime} \in W$, then $e=e^{\prime}$. 
For $x \in \mathcal{R}$, it makes sense to talk about the two-sided orbit $B x B \subset M$, because $T \subset B$. Remarkably, there is an analogue of the Bruhat decomposition for reductive monoids, namely,

$$
M=\bigsqcup_{r \in \mathcal{R}} B r B
$$

See [R8, Theorem 8.8] for more details.

On the Renner monoid $\mathcal{R}$ we define the Bruhat-Chevalley order by

$$
x \leq y \text { if and only if } B x B \subseteq \overline{B y B} .
$$

The induced poset structure on $W$ coincides with the (classical) Bruhat-Chevalley order on $W$. This order on $\mathcal{R}$ extends the order on $E(\bar{T})$ defined a few paragraphs above. See [R8, Section 8.6] for details.

The decomposition of $M$ into $G \times G$-orbits has its analogue on $\mathcal{R}$, namely,

$$
\mathcal{R}=\bigsqcup_{e \in \Lambda} W e W
$$

a decomposition into $W \times W$-orbits.

We denote by $\mathcal{R}_{k}$ the set of elements of rank $k$ in $\mathcal{R}$, that is,

$$
\mathcal{R}_{k}=\{x \in \mathcal{R} \mid \operatorname{dim} T x=k\} .
$$

Analogously, one defines $\Lambda_{k} \subset \Lambda$ and $E_{k} \subset E(\bar{T})$.

Finally, we conclude this review by introducing some important subgroups of $G \subset M$. For $e \in E(M)$, define

$$
P_{e}:=\{g \in G \mid g e=e g e\} \text { and } P_{e}^{-}:=\{g \in G \mid e g=e g e\} .
$$

It is known that $P_{e}$ and $P_{e}^{-}$are opposite parabolic subgroups of $G$, with common Levi subgroup $C_{G}(e)=\{g \in G \mid g e=e g\}$, the centralizer of $e$ in $G$. In particular $C_{G}(e)$ is connected and reductive. Moreover, the unipotent radical $U_{e}$ of $P_{e}$ (resp. $U_{e}^{-}$of $P_{e}^{-}$) satisfies $U_{e} \cdot e=\{e\}$ (resp. $e \cdot U_{e}^{-}=\{e\}$ ). See [R8, Theorem 4.5]. If $e \in E(\bar{T})$, then we write $C_{W}(e)$ for the centralizer of $e$ in $W$. In this case, $C_{W}(e)$ is the Weyl group of $T$ in $C_{G}(e)$ (see [R2, Section 9.5]).

1.2. Projective group embeddings. Let $M$ be a reductive monoid with zero and unit group $G$. Let $\epsilon: \mathbb{G}_{m} \rightarrow T$ be a central one-parameter subgroup, with image $Z$, such that $\lim _{t \rightarrow 0} \epsilon(t)=0$ [Br6, Lemma 1.1.1]. We denote by $\mathbb{P}_{\epsilon}(M)$ the projective group embedding $(M \backslash\{0\}) / Z$. Recall that all projective embeddings of connected reductive groups are obtained by this procedure [R3]. If $M$ is semisimple (i.e. $G$ has a one-dimensional center), then $\epsilon$ is essentially unique, and we write $\mathbb{P}(M)$ instead of $\mathbb{P}_{\epsilon}(M)$.

Example 1.1. Let $G_{0}$ be a semisimple algebraic group and let $\rho: G_{0} \rightarrow \operatorname{End}(V)$ be a finite dimensional irreducible representation of $G_{0}$. Define $Y_{\rho}$ to be the Zariski closure of $G=\left[\rho\left(G_{0}\right)\right]$ in $\mathbb{P}(\operatorname{End}(V))$, the projective space associated with $\operatorname{End}(V)$. Finally, let $X_{\rho}$ be the normalization of $Y_{\rho}$. By definition, $X_{\rho}$ is a projective embedding of $G$. Notice that $M_{\rho}$, the normalization of the Zariski closure of $\mathbb{k}^{*} \rho\left(G_{0}\right)$ in $\operatorname{End}(V)$, is a semisimple monoid whose group of units is $\mathbb{k}^{*} \rho\left(G_{0}\right)$. Embeddings of this kind are studied in more detail in Section 4. 
It follows from Subsection 1.1 that the $G \times G$-orbits in $\mathbb{P}_{\epsilon}(M)$ are indexed by $\Lambda \backslash\{0\}$. Similarly, the $B \times B$-orbits of $\mathbb{P}_{\epsilon}(M)$ are indexed by $\mathcal{R} \backslash\{0\}$. With these identifications, the set of closed $G \times G$-orbits of $\mathbb{P}_{\epsilon}(M)$ corresponds to $\Lambda_{1}$. Next is a structural description of the $G \times G$-orbits in $\mathbb{P}_{\epsilon}(M)$.

Proposition 1.2. Let $X=\mathbb{P}_{\epsilon}(M)$ be a projective group embedding. Let $e \in \Lambda$. Let $H_{e}$ denote the $G \times G$-stabilizer of $[e] \in X$. Then there is a fibration sequence

$$
e C_{G}(e) / \mathbb{G}_{m} \longrightarrow(G \times G) / H_{e} \stackrel{\pi}{\longrightarrow} G / P_{e} \times G / P_{e}^{-} .
$$

In particular, if $e \in \Lambda_{1}$, then

$$
(G \times G) / H_{e} \simeq G / P_{e} \times G / P_{e}^{-},
$$

for, in this case, $e M e \simeq \mathbb{k}, e C_{G}(e) \simeq e \times \mathbb{G}_{m}$ and $P_{e} \cdot e=\mathbb{G}_{m} \cdot e$.

Proof. Note that $H_{e}$ is contained in the subgroup $P_{e} \times P_{e}^{-}$. To see this, let $(g, h) \in$ $H_{e}$. Then $g e h^{-1}=e z$, for some $z \in Z \simeq \mathbb{G}_{m}$. That is, egeh ${ }^{-1} z^{-1}=e^{2}$, but $e$ is an idempotent, so $e g e h^{-1} z^{-1}=e$. The latter yields $e g e=e z h$, and the right hand side equals $g e$, by assumption. Thus ege $=g e$. Analogously, one checks $e h=e h e$. Since $H_{e} \subset P_{e} \times P_{e}^{-}$, there is a natural map of homogeneous spaces $\pi$ : $(G \times G) / H_{e} \rightarrow G / P_{e} \times G / P_{e}^{-}$, whose fiber is $\left(P_{e} \times P_{e}^{-}\right) / H_{e}$. We claim that this fibre is isomorphic to $e C_{G}(e) / e Z \simeq e C_{G}(e) / \mathbb{G}_{m}$. Indeed, first recall that $P_{e}=C_{G}(e) U_{e}$ and $P_{e}^{-}=C_{G}(e) U_{e}^{-}$. Moreover, $U_{e} \cdot e=\{e\}$ and $e \cdot U_{e}^{-}=\{e\}$ (Subsection 1.1). Also, by [R8, Theorem $4.8(\mathrm{a})], e C_{G}(e)$ is a connected reductive group with unit $e$. Hence the map $p_{e}: P_{e} \times P_{e}^{-} \rightarrow\left(C_{G}(e) e / Z e\right) \times\left(e C_{G}(e) / e Z\right)$, sending $(g, h)$ to $([g e],[e h])$ is a well-defined surjective group homomorphism (cf. [P2, Proof of Proposition 2.2]). By considering the corresponding morphism of Lie algebras, one easily checks that the differential of $p_{e}$ at $(1,1)$ is surjective; that is, $p_{e}$ is separable $\mathrm{Sp}$. Theorem 4.3.7 (iii)]. Now observe that $p_{e}$ maps $H_{e}$ onto $\operatorname{diag}\left(e C_{G}(e) / e Z\right)$. In consequence, $P_{e} \times P_{e}^{-} / H_{e} \simeq\left(e C_{G}(e) / e Z\right) \times\left(e C_{G}(e) / e Z\right) / \operatorname{diag}\left(e C_{G}(e) / e Z\right) \simeq e C_{G}(e) / e Z$. For the last assertion of the Proposition, notice that $e \in \Lambda_{1}$ is a minimal idempotent, so $e M e$ is a reductive monoid isomorphic to $\mathbb{k}$, with unit group $e C_{G}(e) \simeq \mathbb{G}_{m}[\mathrm{R} 8$, Theorem 4.8].

Finally, associated to $\mathbb{P}_{\epsilon}(M)$, there is a projective torus embedding of $T / Z$, namely, $\mathbb{P}_{\epsilon}(\bar{T})=[\bar{T} \backslash\{0\}] / Z$. By construction, $\mathbb{P}_{\epsilon}(\bar{T})$ is a (normal) projective toric variety contained in $\mathbb{P}_{\epsilon}(M)$. Notice that the $T$-orbit structure of $\mathbb{P}_{\epsilon}(\bar{T})$ is governed by $E(\bar{T}) \backslash\{0\}$. This toric variety will play an important role in Sections 3 and 4 .

\section{2. $G K M$ DATA OF PROJECTIVE GROUP EMBEDDINGS}

We maintain the notation from Section 1 . Let $X=\mathbb{P}_{\epsilon}(M)$ be a projective group embedding. In this section we show that $X$ is $T \times T$-skeletal, i.e. $X$ has only finitely many $T \times T$-fixed points and $T \times T$-invariant curves. Furthermore, for each $T \times T$-invariant curve of $X$ we obtain explicitly the associated character of $T \times T$. We write down this GKM data in terms of the combinatorial invariants of $M$. The calculations do not depend on any special property of $M$ or $X$.

Our initial task is to identify the following two sets.

(1) $\{x \in M \mid \operatorname{dim} T x T=1\}$.

(2) $\{x \in M \mid \operatorname{dim} T x T=2\}$.

The first class will determine the set $X^{T \times T}$, whereas the second one will determine the set of $T \times T$-invariant curves in $X$. 
2.1. Fixed Points. We identify $\mathcal{R}_{1}$ with its image in $X=\mathbb{P}_{\epsilon}(M)$, and consider it as a subset of $X$. Also, $Z \subseteq T$ is the given attractive one-parameter subgroup in the center of $G$.

Theorem 2.1. The subsets $\mathcal{R}_{1}$ and $X^{T \times T}$ are equal. In particular, there is only a finite number of $T \times T$-fixed points in $X$.

Proof. The set $X^{T \times T}$ corresponds to $\{x \in M \mid \operatorname{dim}(T x T)=1\}$. Note that if $\operatorname{dim}(T x)=1$, then $T x=Z x$. Similarly, if $\operatorname{dim}(x T)=1$, then $x T=Z x$. These remarks, together with the fact that $T x \cup x T \subseteq T x T$, yield the equality

$$
\{x \in M \mid \operatorname{dim}(T x T)=1\}=\{x \in M \mid T x=x T \text { and } \operatorname{dim}(T x)=1\} .
$$

The latter set is precisely $\mathcal{R}_{1}$.

\subsection{Invariant Curves.}

Proposition 2.2. Let $x \in M$ and assume that $x \neq 0$. Then the following are equivalent.

(1) $\operatorname{dim} T x T=2$.

(2) Either $\operatorname{dim}(x T)=2$ and $T x \subseteq x T, x T=T x T$; or $\operatorname{dim}(T x)=2$ and $x T \subseteq T x, T x=T x T$; or $\operatorname{dim}(T x T)=2$ and $T x=x T=T x T$.

Proof. It suffices to check that (1) implies (2), for the other direction is obvious. So assume that (1) holds. Now $T x \cup x T \subseteq T x T$. If $\operatorname{dim}(T x)=\operatorname{dim}(x T)=1$, then $T x=Z x=x T$. But then $\operatorname{dim}(T x T)=1$, a contradiction. Hence at least one of $T x$ or $x T$ is two-dimensional. If $\operatorname{dim}(T x)=2$, then $T x \subseteq T x T$ yet they have the same dimension. Thus $T x=T x T$. If $\operatorname{dim}(x T)=2$, then we end up with $x T=T x T$.

Corollary 2.3. Exactly one of the following assertions is true for $x \in M$ such that $\operatorname{dim}(T x T)=2$.

(1) $x T \subset T x=T x T$ and $\operatorname{dim}(x T)=1$.

(2) $T x \subset x T=T x T$ and $\operatorname{dim}(T x)=1$.

(3) $x T=T x=T x T$.

The following result, due to Renner [R4, Lemma 3.3], will be needed in the sequel.

Lemma 2.4. Let $M$ be a reductive monoid with zero and unit group $G$. Let $T \subseteq G$ be a maximal torus. Choose a central one-parameter subgroup $\epsilon: \mathbb{G}_{m} \rightarrow G$, with image $Z$, that converges to 0 . Then

$$
\{x \in M \backslash\{0\} \mid Z x=T x\}=\bigsqcup_{e \in E_{1}} e G .
$$

Consequently, if $X=\mathbb{P}_{\epsilon}(M)$ and $e X=(e M \backslash\{0\}) / Z \simeq e G / Z$ then

$$
X^{T}=\bigsqcup_{e \in E_{1}} e X
$$

for the action $T \times X \rightarrow X$ given by $(t,[x]) \rightsquigarrow[t x]$. Similar results hold for the right action $([x], t) \rightsquigarrow[x t]$ of $T$ on $X$.

Theorem 2.5. There are only three types of closed irreducible $T \times T$-curves in $X$, namely:

(1) $\overline{U_{\alpha}[e w]}$, where $e \in E_{1}, s_{\alpha} \notin C_{W}(e)$ and $w \in W$ (fixed pointwise by $T$ on the right). 
(2) $\overline{[w e] U_{\alpha}}$, where $e \in E_{1}, s_{\alpha} \notin C_{W}(e)$ and $w \in W$ (fixed pointwise by $T$ on the left).

(3) $\overline{T[x]}=\overline{[x] T}=\overline{T[x] T}$, where $x \in \mathcal{R}_{2}=\{x \in \mathcal{R} \mid \operatorname{dim}(T x)=2\}$.

In particular, the number of $T \times T$-invariant curves in $X$ is finite.

Proof. Keeping the numeration of Corollary 2.3, we know that the $T \times T$-curves of $X$ fall into three classes. The first two types correspond, as Lemma 2.4 dictates, to curves that are fixed pointwise by $T$ on either the left or the right. The former collection lies on $X^{T}=\bigsqcup_{e \in E_{1}} e G / Z$. Moreover, due to the Bruhat decomposition, for each $e \in E_{1}$ the following identity holds

$$
e G / Z=G / P_{e}^{-}=\bigsqcup_{r \in e W}[r] B_{u},
$$

where $B_{u}$ is the unipotent radical of $B$.

Our task is to find all the $T$-curves of $e G / Z$, where $e$ varies over all the rank-one idempotents of $\bar{T}$. So fix an idempotent $e \in E_{1}$. It follows from the results of Carrell ([C], [CK, Lemma 2.2]), that the $T$-curves of $e G / Z$ are of the form $[r] U_{\alpha}$, for some root $\alpha$ such that $s_{\alpha} \notin C_{W}(f)$ and $f=w^{-1} e w$ (here, $r=e w=w f$ ). Indeed, since $f$ is a rank-one idempotent, then $s_{\alpha} \in C_{W}(f)$ if and only if $U_{\alpha} f=f U_{\alpha}=\{f\}$ [R2, Lemma 5.1]. Because there is no essential difference between $e$ and $f$, we conclude that a $T \times T$-curve, $T x T$, is fixed pointwise on the left by $T$ if and only if $T x T=w f U_{\alpha}$, where $\alpha \notin C_{W}(f), f \in E_{1}$, and $w \in W$. A similar argument disposes of the case when a $T \times T$-curve is fixed pointwise by $T$ on the right.

Finally, if $T x=x T=T x T$ and $\operatorname{dim}(T x)=2$, then $x \in \mathcal{R}_{2}$. Identifying $x \in \mathcal{R}_{2}$ with its image $[x]$ in $X$, it is clear that $T[x] T$ is a $T \times T$-curve in $X$.

Let us state Theorem 2.1 and Theorem 2.5 in a more compact form.

Theorem 2.6. Let $X=\mathbb{P}_{\epsilon}(M)$ be a projective group embedding. Then, for its natural $T \times T$-action, $X$ is $T \times T$-skeletal.

2.3. Classification of $G K M$-curves. Let $X=\mathbb{P}_{\epsilon}(M)$ be a projective group embedding. From Theorem 2.5, we also know that there are three types of $T \times T$-curves in $X$ :

(1) Curves that are fixed pointwise by $T$ on the right: $\overline{U_{\alpha}[e w]}, e \in E_{1}, s_{\alpha} \notin$ $C_{W}(e)$, and $w \in W$.

(2) Curves that are fixed pointwise by $T$ on the left: $\overline{[w e] U_{\alpha}}, e \in E_{1}, s_{\alpha} \notin$ $C_{W}(e)$, and $w \in W$.

(3) $\overline{T[x]}=\overline{[x] T}=\overline{T[x] T}$ where $x \in \mathcal{R}_{2}=\{x \in \mathcal{R} \mid \operatorname{dim}(T x)=2\}$.

But which pair of fixed points, i.e. elements of $\mathcal{R}_{1}$, is joined by each of these curves? Preserving the given order, we obtain

(1) ew and $s_{\alpha} e w$

(2) we and $w_{e} s_{\alpha}$

(3) The two elements $r, s \in \mathcal{R}_{1}$ such that $r, s \in \overline{T x T}$.

Theorem 2.7. The set of $T \times T$ - curves in $X=\mathbb{P}_{\epsilon}(M)$ is identified as follows, by pairs of $T \times T$-fixed points. Here Re $f(W)$ refers to the set of reflections of $W$, the ordering on $\mathcal{R}$ is the Bruhat-Chevalley order (Section 1.1).

(1) $\left\{(x, s x) \mid x \in \mathcal{R}_{1}, s \in \operatorname{Ref}(W)\right.$ and $\left.x>s x\right\}$.

(2) $\left\{(x, x s) \mid x \in \mathcal{R}_{1}, s \in \operatorname{Ref}(W)\right.$ and $\left.x>x s\right\}$. 
(3) $\mathcal{R}_{2} \cong\left\{A \subseteq \mathcal{R}_{1}|| A \mid=2\right.$ and $A=\{e x, f x\}$ for some $e, f \in E_{1}$ and some $x \in$ $\left.\mathcal{R}_{2}\right\}$.

Proof. Assertions (1) and (2) follow from the fact that if $x \neq s x$ and $s \in \operatorname{Ref}(W)$, then either $x<s x$ or else $s x<x$. Indeed, first write $x$ in normal form [R8, Definition 8.34], that is, write $x=v e u$, where $e \in \Lambda_{1}$ and $v, u$ are minimal length coset representatives in $W / C_{W}(e)$, then use [ $\mathbf{R} 8$, Corollary 8.35]. For (3) we proceed as follows. Recall that any $x \in \mathcal{R}_{2}$ can be written as $x=f u$, where $f \in E_{2}$ is a rank-two idempotent, and $u \in W$. Since $u$ is invertible, it is enough to prove the statement for $x=f$. Now notice that $(f \bar{T} \backslash\{0\}) / Z$ is isomorphic to $\mathbb{P}^{1}$ (see e.g. Br4, Corollary 1.4.1]). Thus there are exactly two fixed points, they correspond to the unique rank-one idempotents $e, e^{\prime} \in E_{1}$ such that ef $\neq 0$ and $e^{\prime} f \neq 0$. These two idempotents determine $f$ uniquely (to see this, simply represent $\bar{T}$ as a closed submonoid of End $\left(\mathbb{k}^{n}\right)$ consisting of diagonal matrices). Finally, note that the pair $\left(e, e^{\prime}\right)$ cannot be any of the ones indexed in type (1) or (2) above, for otherwise either $e^{\prime}=s e$ or $e^{\prime}=e s$, where $s$ is a reflection and $s \notin C_{W}(e)$. But then $s e \in E_{1}$ or $e s \in E_{1}$, and any of these would imply $s e=e s=e$, by the uniqueness of the decomposition in the Renner monoid, a contradiction.

Notice that the description in (3) above is just a convenient, indirect way of identifying the elements of $\mathcal{R}_{2}$ as pairs of $T \times T$ - fixed points. Notice also that, for each $x \in \mathcal{R}_{2}$, there are exactly two idempotents $e, f \in E_{1}$ such that $e x \neq 0$ and $f x \neq 0$.

Any $T \times T$-fixed point of $X$ is contained in a closed $G \times G$-orbit (indeed, recall that $\mathcal{R}_{1}=\bigsqcup_{e \in \Lambda_{1}} W e W$, and for each $e \in \Lambda_{1}$, the orbit $W e W$ is identifiable with the $T \times T$-fixed points of the complete homogeneous space $G[e] G)$. The curves identified in (1) and (2) of Theorem 2.7 are the ones that are contained in closed $G \times G$-orbits. The curves identified in (3) of Theorem 2.7 are those that are not contained in any closed $G \times G$-orbit. As in [Br3], these curves are further separated into whether or not the corresponding fixed points are in the same closed $G \times G$ orbit (Lemma 2.12). This distinction will become relevant in the next section when we identify the character associated with each $T \times T$-curve of type (3).

Example 2.8. We illustrate Theorem 2.7 with the example $M=M_{n}(\mathbb{k})$. Let $E_{i, j}$ denote an elementary matrix. We then obtain (with the ordering as in Theorem 2.7)

(1) $\left\{\left(E_{i, j}, E_{k, j}\right) \mid i \neq k\right\}$.

(2) $\left\{\left(E_{i, j}, E_{i, k}\right) \mid j \neq k\right\}$.

(3) $\left\{\left(E_{i, j}, E_{k, l}\right) \mid i \neq k\right.$ and $\left.j \neq l\right\}$.

In each case the associated curve is the $T \times T$-orbit of the sum of the given pair of elementary matrices. In case (1) the two elementary matrices are in the same row. In case (2) the two elementary matrices are in the same column. Case (3) determines the remaining cases.

2.4. The Associated Characters. Let us briefly recall how a character is associated with a $T$-stable curve. Let $X$ be a complete $T$-variety and let $C$ be a $T$-stable irreducible curve of $X$, which is not fixed pointwise by $T$. Let $\pi: \tilde{C} \rightarrow C$ be the (T-equivariant) normalization. Then $\tilde{C}$ is isomorphic to $\mathbb{P}^{1}$. Denote by $0, \infty$ the two fixed points of $T$ in $\tilde{C}$, and denote by $x_{0}, x_{\infty}$ their corresponding images via $\pi$. 
Then $\tilde{C} \backslash\{0, \infty\}=C \backslash\left\{x_{0}, x_{\infty}\right\}$ identifies to $\mathbb{k}^{*}$, where $T$ acts on $\tilde{C} \backslash\{0, \infty\}$ via a unique character $\chi$ (when interchanging 0 and $\infty$, one replaces $\chi$ by $\chi^{-1}$ ).

In this Subsection we identify the character $\theta_{x}=\left(\lambda_{x}, \rho_{x}\right)$ of $T \times T$ associated with a $T \times T$-curve $\overline{T[x] T}$ in $X=\mathbb{P}_{\epsilon}(M)$. As discussed previously (Theorems 2.5 and 2.7), there are three different types of $T \times T$-curves in $X$. For the curves of type (1) and (2) the whole issue reduces to the well-documented situation of [C] and $\mathrm{CK}$, Lemma 2.2], since these curves are contained in the closed $G \times G$-orbits of $X$ (such closed orbits are complete homogeneous spaces, Proposition 1.2). Hence,

(1) For a $T \times T$-curve $\overline{U_{\alpha}[e w]}$, with $e \in E_{1}, s_{\alpha} \notin C_{W}(e)$, and $w \in W$, the associated character is $(\alpha, 1)$.

(2) For a $T \times T$-curve $\overline{[w e] U_{\alpha}}$, with $e \in E_{1}, s_{\alpha} \notin C_{W}(e)$, and $w \in W$, the associated character is $(1, \alpha)$.

Consequently, we only need to focus on the curves of type (3), that is, those of the form $\overline{T[x] T}$, with $x \in \mathcal{R}_{2}$.

So let $x \in \mathcal{R}_{2}$. Since we are working on the monoid level, the initial step in our discussion is to calculate the map

$$
m_{x}: T \times T \rightarrow T x T, \quad(s, t) \rightsquigarrow s x t^{-1} .
$$

We then compose $m_{x}$ with the canonical map $\pi_{x}: T x T \rightarrow T x T / Z \cong \mathbb{G}_{m}$ to obtain

$$
\theta_{x}=\pi_{x} \circ m_{x}
$$

where $Z \subseteq G$ is the given central, attractive, 1-parameter subgroup of the unit group $G$ of $M$. Notice that $\theta_{x}$ depends on the choice of group isomorphism $T x T / Z \cong \mathbb{G}_{m}$. The other isomorphism $T x T / Z \cong \mathbb{G}_{m}$ yields $\theta_{x}^{-1}$. In the calculation of $\theta_{x}$ it is important to keep track of this ambiguity. It is also useful to consider the map

$$
\tau_{x}: T \rightarrow T x, t \rightsquigarrow t x
$$

and the character $\lambda_{x}=\pi_{x} \circ \tau_{x}$. Notice that $T x T=T x$, so we wish to express $\theta_{x}: T \times T \rightarrow \mathbb{G}_{m}$ as a composition

$$
T \times T \rightarrow T \times T \rightarrow T \rightarrow T x \rightarrow \mathbb{G}_{m}
$$

involving the $W \times W$-action on $T \times T$, the multiplication $T \times T \rightarrow T$, and these other quantities: $\tau_{x}, \pi_{x}, \lambda_{x}$. We also assess how the $W \times W$-action on $\mathcal{R}_{2}$ affects the characters associated to the curves $\overline{T[x] T}, x \in \mathcal{R}_{2}$. This will effectively reduce the calculation of $\theta_{x}$, with $x \in \mathcal{R}_{2}$, to calculating $\theta_{x}$ for a set of representatives of the $W \times W$-orbits of $\mathcal{R}_{2}$.

Write $x$ as $x=f u=u g$, where $u \in W$ and $f, g \in E_{2}$. An elementary calculation yields that

$$
m_{x}: T \times T \rightarrow T x T=x T, \quad(s, t) \rightsquigarrow s x t^{-1}
$$

is given by $m_{x}(s, t)=s\left(t^{u}\right)^{-1} x$ where, by definition, $t^{u}=u t u^{-1}$. Recall that $\lambda_{x}=\pi_{x} \circ \tau_{x}$, where $\tau_{x}: T \rightarrow T x, t \rightsquigarrow t x$, and $\pi_{x}: T x T \rightarrow T x T / Z \cong \mathbb{G}_{m}$.

Lemma 2.9. Write $\theta_{x}=\left(\lambda_{x}, \rho_{x}\right) \in \Xi \oplus \Xi$, where $\Xi \oplus \Xi$ is the character group of $T \times T$. Then

(1) $\lambda_{x}=\lambda_{f}$.

(2) $\rho_{x}=\lambda_{g}^{-1}=\lambda_{f}^{-1} \circ \operatorname{int}(u)$, where $\operatorname{int}(u)(t)=u t u^{-1}$. 
Proof. Consider $m: T \times T \rightarrow T f, \quad(s, t) \rightsquigarrow s\left(t^{u}\right)^{-1} f$. Then $m(s, t) \in Z f$ if and only if $m_{x}(s, t) \in Z x$. So $k e r\left(\pi_{f} \circ m\right)=\operatorname{ker}\left(\pi_{x} \circ m_{x}\right)$. Thus $\lambda_{x}=\lambda_{f}$ and $\rho_{x}=\lambda_{f}^{-1} \circ$ $\operatorname{int}(u)$. On the other hand, $m$ is also the product of $(s, 1) \rightsquigarrow s f$ and $(1, t) \rightsquigarrow\left(t^{u}\right)^{-1} f$. The first of these is $\lambda_{f}$ and the second of these is $\lambda_{f}^{-1} \circ$ int $(u)$. Clearly, $\left(t^{u}\right)^{-1} f \in Z f$ if and only if $t^{-1} g \in Z g$, because $u g u^{-1}=f$. Thus $\operatorname{ker}\left(\lambda_{x}^{-1} \circ \operatorname{int}(u)\right)=\operatorname{ker}\left(\lambda_{g}^{-1}\right)$. We conclude that $\theta_{x}=\left(\lambda_{x}, \rho_{x}\right)=\left(\lambda_{f}, \lambda_{g}^{-1}\right)=\left(\lambda_{f}, \lambda_{f}^{-1} \circ \operatorname{int}(u)\right)$.

It is worth noting that we can also write $m_{x}$ as $m_{x}: T \times T \rightarrow T x T=x T$, $m_{x}(s, t)=s x t^{-1}=x s^{u^{-1}} t^{-1}$ (notice that $x$ now appears on the left). The resulting calculation then yields $\theta_{x}=\left(\lambda_{x}, \rho_{x}\right)=\left(\lambda_{g} \circ \operatorname{int}\left(u^{-1}\right), \lambda_{g}^{-1}\right)=\left(\lambda_{f}, \lambda_{g}^{-1}\right)$.

Observe that either $\theta_{x}=\left(\lambda_{x}, \lambda_{x}^{-1} \circ \operatorname{int}(u)\right)$ or $\theta_{x}=\left(\lambda_{x}^{-1}, \lambda_{x} \circ\right.$ int $\left.(u)\right)$ depending on the orientation. Also, it follows from Lemma 2.9 that if $f, g \in E_{2}$ are conjugate, i.e. $f=w g w^{-1}$ for some $w \in W$, then $\lambda_{f}=\lambda_{g} \circ \operatorname{int}\left(w^{-1}\right)$.

Lemma 2.10. Let $x \in \mathcal{R}_{2}$. Let $\theta_{x}=\left(\lambda_{x}, \rho_{x}\right)$ be the associated character, as in Lemma 2.9.

(1) If $y=x w$, where $w \in W$, then $\theta_{y}=\left(\lambda_{x}, \rho_{x} \circ\right.$ int $\left.(w)\right)$.

(2) If $y=w x$, where $w \in W$, then $\theta_{y}=\left(\lambda_{x} \circ \operatorname{int}\left(w^{-1}\right), \rho_{x}\right)$.

Proof. As before, write $x=f u$, with $f \in E_{2}$ and $u \in W$. If $y=x w$, then $y=f u w$, and so Lemma 2.9, yields $\theta_{y}=\left(\lambda_{f}, \lambda_{f}^{-1} \circ \operatorname{int}(u w)\right)=\left(\lambda_{f}, \lambda_{f}^{-1} \circ \operatorname{int}(u) \circ \operatorname{int}(w)\right)$. Thus $\theta_{y}=\left(\lambda_{x}, \rho_{x} \circ \operatorname{int}(w)\right)$. On the other hand, if $y=w x$, then $y=w f u=f^{\prime} w u$, where $f^{\prime}=w f w^{-1}$. Once again, Lemma 2.9 implies $\theta_{y}=\left(\lambda_{f^{\prime}}, \lambda_{f^{\prime}}^{-1} \circ \operatorname{int}(w u)\right)$. Moreover, $\lambda_{f^{\prime}}=\lambda_{f} \circ \operatorname{int}\left(w^{-1}\right)$. Thus $\theta_{y}=\left(\lambda_{x} \circ \operatorname{int}\left(w^{-1}\right), \rho_{x}\right)$.

Next we state a slight variation on a result of Putcha $\mathrm{Pu}$, Proposition 10.9].

Lemma 2.11. Let $f \in E_{2}$. Then either there is a unique $s \in C_{W}(f)$ such that $s f=f s \neq f$, or every $s \in C_{W}(f)$ satisfies $s f=f s=f$. In the former case, $s$ is a reflection, denoted $s_{\alpha_{f}}$. Moreover, $\lambda_{f}=\alpha_{f}$, a root of $(G, T)$.

Proof. Since $C_{W}(f)=\left\langle s_{\alpha} \mid \alpha \in \Delta, s_{\alpha} f=f s_{\alpha}\right\rangle$ [Pu, Lemma 10.15], the first two assertions follow directly from $[\mathrm{Pu}$, Proposition 10.9]. Thus, assuming there is a unique reflection $s:=s_{\alpha_{f}} \in C_{W}(f)$ with the property that $s f=f s \neq f$, it remains to show that $\lambda_{f}=\alpha_{f}$. In fact, since the map $T \rightarrow T f$ is $s$-equivariant and $s$ acts trivially on $\operatorname{Ker}(T \rightarrow T f)[\mathrm{Pu}$, Corollary 10.11], it suffices to check that $\lambda_{f}$ and $\alpha_{f}$ agree on $T f$. For this, consider the inner transformation $\operatorname{int}(s)$ : $f \bar{T} \rightarrow f \bar{T}, f x \mapsto f s x s^{-1}$. Let us examine the automorphism $\sigma$ induced by int $(s)$ on $f \bar{T}-\{0\} / Z \simeq \mathbb{P}^{1}$. Recall that there are exactly two rank-one idempotents $f_{1}$ and $f_{2}$ below $f$. Denote by 0 and $\infty$, respectively, their classes in the orbit space $f \bar{T}-\{0\} / Z$. Also, since $f$ is the identity element of the reductive monoid $f \bar{T}$, let us denote its class on $\mathbb{P}^{1}$ by 1 . Because $\left(s f_{i} s^{-1}\right) \cdot f=s f_{i} s^{-1}$ for $i=1,2$, it is clear that $\sigma$ permutes the points 0 and $\infty$. So either $\sigma(0)=0$ and $\sigma(\infty)=\infty$ or else $\sigma(0)=\infty$ and $\sigma(\infty)=0$. Moreover, $\sigma(1)=1$ in either case, because $\sigma$ restricts to an algebraic automorphism of $\mathbb{G}_{m} \simeq T f / Z=\mathbb{P}^{1} \backslash\{0, \infty\}$. Hence, as a Möbius transformation, $\sigma$ is either $z \mapsto z$ or $z \mapsto z^{-1}$. The former is clearly impossible because, by assumption, $s f=f s \neq f$. Therefore, by looking at the commutative 
diagram

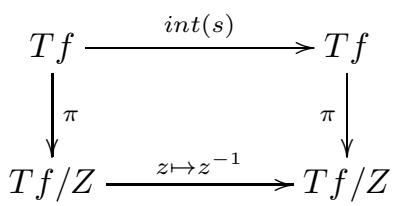

we conclude that $s$, when restricted to $T f$, is also a reflection. Clearly, $\alpha_{f}$ is uniquely determined by the commutative diagram above, thus $\lambda_{f}=\alpha_{f}$.

Lemma 2.12. Let $x \in \mathcal{R}_{2}$, and write $x=f u$, with $f \in E_{2}$ and $u \in W$. The following are equivalent.

(1) There is a unique reflection $s_{\alpha_{f}}$ such that $s_{\alpha_{f}} f=f s_{\alpha_{f}} \neq f$.

(2) The two $T \times T$-fixed points of $\overline{T[x] T} \subset X$ are in the same $W \times W$-orbit.

Proof. Let $x \in \mathcal{R}_{2}$ and let $a, b \in \overline{T x T}$ represent the two $T \times T$-fixed points in $\overline{T[x] T}$. Then $a=f_{1} x=f_{1} u$ and $b=f_{2} x=f_{2} u$ where $f_{1}, f_{2}$ are the two rank-one idempotents below $f$. Assume (1) holds. Set $s:=s_{\alpha_{f}}$. Notice that $s f_{1} s=f_{2}$ since $s f=f s \neq f$. So by setting $t:=u^{-1} s u$, one gets $b=s a t$ and $a=s b t$.

Now let $x=f u \in \mathcal{R}_{2}$ and assume that $f_{1} x=f_{1} u$ and $f_{2} x=f_{2} u$ are in the same $W \times W$-orbit. Then $f_{1}$ and $f_{2}$ are in the same $W \times W$-orbit. This implies that $f_{1}$ and $f_{2}$ are conjugate (Subsection 1.1). Furthermore, $\mathrm{Pu}$, Corollary 8.9 and Proposition 10.9] asserts that $f_{1}$ and $f_{2}$ are conjugate by an element $s \in C_{W}(f)=$ $\{v \in W \mid v f=f v\}$. It follows that $s f=f s \neq f$ (for otherwise, $s f_{1} s^{-1}=f_{1}$ but $\left.f_{1} \neq f_{2}\right)$. Thus $s=s_{\alpha_{f}}$ (Lemma 2.11).

The next result follows immediately from Lemmas 2.9, 2.10 and 2.11.

Lemma 2.13. Let $x \in \mathcal{R}_{2}$. Write $x=f u$ and assume there is a (unique) reflection $s_{\alpha_{f}}$ such that $s_{\alpha_{f}} f=f s_{\alpha_{f}} \neq f$. Then $\lambda_{f}=\alpha_{f}$ and $\lambda_{f} \circ \operatorname{int}\left(s_{\alpha_{f}}\right)=\lambda_{f}^{-1}$. In particular, the components of $\theta_{x}=\left(\lambda_{x}, \rho_{x}\right)$ are roots of $(G, T)$, namely,

$$
\theta_{x}=\left(\lambda_{x}, \rho_{x}\right)=\left(\alpha_{f}, \alpha_{f}^{-1} \circ \operatorname{int}(u)\right) .
$$

Moreover, if $y=s_{\alpha_{f}} x$, then $\theta_{y}=\left(\lambda_{x}^{-1}, \rho_{x}\right)=\left(\alpha_{f}^{-1}, \alpha_{f}^{-1} \circ\right.$ int $\left.(u)\right)$.

Example 2.14. Let $M=M_{n}(\mathbb{k})$ and let $T$ be the set of invertible, diagonal matrices. One checks that

$$
\mathcal{R}_{2}=\left\{E_{i, j}+E_{k, l} \mid i \neq k \text { and } j \neq l\right\} .
$$

where $E_{i, j}$ denotes the elementary matrix with a one in the $(i, j)$-position and zeros elsewhere. Let $\underline{s}=\left(s_{1}, \ldots, s_{n}\right) \in T$ denote the obvious diagonal matrix. A simple calculation yields that, for $\underline{s}, \underline{t} \in T$ and $x=E_{i, j}+E_{k, l}$,

$$
\theta_{x}(\underline{s}, \underline{t})=s_{i}^{-1} s_{k} t_{j} t_{l}^{-1} \text {. }
$$

In this case, $x=f u$, with $f=E_{i, i}+E_{k, k}$ and $u=\sigma_{j, i} \sigma_{k, l}$, where $\sigma_{i, j}$ represents the permutation matrix that exchanges the $i$-th and $j$-th columns of the identity matrix. The unique reflection $s_{f}$ satisfying $s_{f} f=f s_{f} \neq f$ is $\sigma_{i, k}$. The corresponding $y=s_{f} x \in \mathcal{R}_{2}$ from Lemma 2.13 is $y=E_{k, j}+E_{i, l}$, Thus,

$$
\theta_{y}(\underline{s}, \underline{t})=s_{i} s_{k}^{-1} t_{j} t_{l}^{-1} \text {. }
$$

In the terminology of Lemma 2.13] $\theta_{x}=\left(\lambda_{x}, \rho_{x}\right)$ where $\lambda_{x}=\alpha_{k, i}=s_{k} s_{i}^{-1}$ and $\rho_{x}=\alpha_{j, l}=t_{j} t_{l}^{-1}$. Similarly, $\lambda_{y}=\alpha_{i, k}$ and $\rho_{y}=\alpha_{j, l}$. 


\section{GKM THEORY OF RATIONALLY SMOOTH PROJECTIVE GROUP EMBEDDINGS}

In this and next section we specialize the results of Section 2 to the case when $\mathbb{k}=\mathbb{C}$ and the group embedding $\mathbb{P}_{\epsilon}(M)$ is rationally smooth.

From now on, in order to consider $S$, the symmetric algebra over $\mathbb{Q}$ of the character group of $T$, we shall write characters in the additive notation, that is, $\left(\chi_{1}+\chi_{2}\right)(t):=\chi_{1}(t) \chi_{2}(t)$.

3.1. GKM theory. We recall some basic facts and nomenclature from GKM. A $T$-variety $X$ is called equivariantly formal if the natural map $H_{T}^{*}(X) \rightarrow H^{*}(X)$ is surjective (i.e. $H^{*}(X)$ is the quotient of $H_{T}^{*}(X)$ by its homogeneous ideal generated by all characters of $T$ ). If $X^{T}$ is finite, then $X$ is equivariantly formal if and only if $X$ has no cohomology in odd degrees Br5, Lemma 1.2].

A $T$-variety is a $G K M$ variety if it is complete, $T$-skeletal, and equivariantly formal.

Theorem 3.1 ([GKM, Theorem 1.2.2]). Let $X$ be a GKM variety, and let $X^{T}=$ $\left\{x_{1}, \ldots, x_{m}\right\}$. Then the pullback

$$
i_{T}^{*}: H_{T}^{*}(X) \rightarrow H_{T}^{*}\left(X^{T}\right)
$$

is injective, and its image is the set of all $\left(f_{1}, \ldots, f_{m}\right) \in S^{m}$ such that $f_{i} \equiv f_{j}$ $\bmod \chi$ whenever $x_{i}, x_{j}$ are connected by an irreducible invariant curve where $T$ acts through the character $\chi$.

For notational purposes, we shall interpret the image of $i_{T}^{*}$ as the set of all maps $\varphi: X^{T} \rightarrow S$ such that $\varphi\left(x_{i}\right) \equiv \varphi\left(x_{j}\right) \bmod \chi$ whenever $x_{i}$ and $x_{j}$ are connected by an irreducible invariant curve where $T$ acts through the character $\chi$.

By a result of Borel [Bo], if $X$ is a $G$-variety, then $H_{G}^{*}(X) \simeq H_{T}^{*}(X)^{W}$.

Lemma 3.2. Let $X$ be a $G$-variety. Suppose that $X$ has no cohomology in odd degrees and that, for the induced $T$-action, $X^{T}$ is finite. Then $H_{G}^{*}(X)$ and $H_{T}^{*}(X)$ are free modules over $S^{W}$ and $S$ respectively, and their ranks satisfy

$$
\operatorname{rank}_{S W} H_{G}^{*}(X)=\operatorname{dim}_{\mathbb{Q}} H^{*}(X)=\operatorname{rank}_{S} H_{T}^{*}(X)=\left|X^{T}\right| .
$$

Proof. By hypothesis, $X$ is equivariantly formal for the induced $T$-action, so $H_{T}^{*}(X)$ is a free $S$-module [Br5, Lemma 1.2]. Now let $F$ be a graded $W$-stable complement of $S_{+} \cdot H_{T}^{*}(X) \subset H_{T}^{*}(X)$, where $S_{+}$is the ideal in $S$ consisting of polynomials without constant term. One checks that $H_{T}^{*}(X) \simeq S \otimes F$. It is known that $S \simeq S^{W} \otimes R$, where $R$ is isomorphic to the regular representation of $W$. So $H_{T}^{*}(X) \simeq S^{W} \otimes R \otimes F$, which implies $H_{G}^{*}(X) \simeq S^{W} \otimes(R \otimes F)^{W}$. But then $(R \otimes F)^{W} \simeq F$, because $R$ is the regular representation of $W$. Thus, $\operatorname{rank}_{S^{W}} H_{G}^{*}(X)=\operatorname{rank}_{S} H_{T}^{*}(X)=$ $\operatorname{dim}_{\mathbb{Q}} H^{*}(X)=\operatorname{dim} F$. Finally, by the Localization Theorem (see e.g. Br5, Lemma 1.1]) we conclude that $\operatorname{rank}_{S} H_{T}^{*}(X)=\left|X^{T}\right|$.

3.2. Rationally smooth group embeddings. Recall that for $e \in E(M)$, the monoid $M_{e}:=\overline{\{g \in G \mid g e=e g=e\}}$ is a reductive monoid with $e$ as its zero element Br6, Corollary 2.3.3]. Next is Renner's characterization of rationally smooth group embeddings.

Theorem $3.3\left([\underline{\mathrm{R} 6})\right.$. Let $\mathbb{P}_{\epsilon}(M)$ be a projective group embedding. Then the following are equivalent.

(1) $\mathbb{P}_{\epsilon}(M)$ is rationally smooth. 
(2) $M \backslash\{0\}$ is rationally smooth.

(3) For any minimal, nonzero, idempotent e of $M, M_{e}$ is rationally smooth.

(4) For any maximal torus $T$ of $G, \bar{T} \backslash\{0\}$ is rationally smooth.

In particular, $\mathbb{P}_{\epsilon}(M)$ is rationally smooth if and only if $\mathbb{P}_{\epsilon}(\bar{T})$ is rationally smooth (a toric variety is rationally smooth if and only if it is simplicial [D]). Also, notice that the condition does not depend on the choice of $Z$.

Example 3.4. Smooth group embeddings are clearly rationally smooth. In particular, so are the projective regular embeddings: smooth projective $G$-embeddings whose closed $G \times G$-orbits are all of the form $G / B \times G / B^{-}$(cf. Proposition 3.13).

Example 3.5. Let $M$ be a semisimple monoid with zero and unit group $G$ of the form $\mathbb{C}^{*} \times G_{0}$, where $G_{0}$ is a simple algebraic group of type $A_{2}, C_{2}$ or $G_{2}$. Then $\mathbb{P}(M)$ is always rationally smooth. Indeed, this follows from Theorem 3.3 since, in this context, $\mathbb{P}(\bar{T})$ is a simplicial toric surface. Note that there are cases when $\mathbb{P}(M)$ has closed $G \times G$-orbits of the form $G / P \times G / P^{-}$, where $P \supsetneq B$ is a parabolic subgroup. Such embeddings, though rationally smooth, are not regular. See R8, Section 5.1] for details.

Example 3.6. Let $G$ be a semisimple adjoint group with Borel subgroup $B$ and maximal torus $T \subset B$. An embedding of $G$ is called simple if it contains a unique closed $G \times G$-orbit. Let $X$ be such an embedding. Then, using the notation from Example 1.1, $X$ is of the form $\mathbb{P}\left(M_{\rho_{\lambda}}\right)$, for some irreducible representation $\rho_{\lambda}$ of $G$, with highest weight $\lambda[\mathrm{R} 6]$. Moreover, the unique closed $G \times G$-orbit of $X$ is the partial flag variety $G / P_{J} \times G / P_{J}^{-}$, where $J=\left\{\alpha \in \Delta \mid s_{\alpha}(\lambda)=\lambda\right\}$. Recall that $P_{J}$ is the standard parabolic subgroup associated to $J$, and $P_{J}^{-}$its opposite. Using Theorem 3.3, Renner has classified all rationally smooth simple embeddings combinatorially, in terms of $J$ and the Dynkin diagram for $G$ R7. In Section 4 we present Renner's list of all possible $J$ 's that yield rationally smooth group embeddings, and discuss the connections to Timashev's description of projective group embeddings via weight polytopes [T2, Section 27].

Next is a justification for the use of GKM theory in the study of rationally smooth group embeddings. It is a consequence of Theorem 2.6] and [G1, Theorem 7.4].

Theorem 3.7. Let $X=\mathbb{P}_{\epsilon}(M)$ be a projective group embedding. If $X$ is rationally smooth, then, for its natural $T \times T$-action, $X$ is a GKM variety.

3.3. The main results. Let $X=\mathbb{P}_{\epsilon}(M)$ be a projective group embedding. Recall that the closed $G \times G$-orbits of $X$ correspond to idempotents $e \in \Lambda_{1}$. Because the $T \times T$-fixed points of $G[e] G \simeq G / P_{e} \times G / P_{e}^{-}$are identifiable with the two-sided orbit $W e W$, equivariant cohomology classes in $H_{T \times T}^{*}(G[e] G)$ correspond, via Theorem 3.1, to functions $\varphi_{e}: W e W \rightarrow S \otimes S$ satisfying the conditions:

(i) $\varphi_{e}(e w) \equiv \varphi_{e}\left(s_{\alpha} e w\right) \bmod (\alpha, 0)$ whenever $s_{\alpha} \notin C_{W}(e)$ and $w \in W$,

(ii) $\varphi_{e}(w e) \equiv \varphi_{e}\left(w_{e} s_{\alpha}\right) \bmod (0, \alpha)$ whenever $s_{\alpha} \notin C_{W}(e)$ and $w \in W$.

Now we state the first major result of this article. For the analogous result in the case of projective regular embeddings, see [Br3, Theorem 3.1.1]. 
Theorem 3.8. Let $X=\mathbb{P}_{\epsilon}(M)$ be a projective group embedding. If $X$ is rationally smooth, then the natural map

$$
H_{T \times T}^{*}(X) \longrightarrow H_{T \times T}^{*}\left(\bigsqcup_{e \in \Lambda_{1}} G[e] G\right)=\bigoplus_{e \in \Lambda_{1}} H_{T \times T}^{*}(G[e] G)
$$

is injective. In fact, its image consists of all tuples $\left(\varphi_{e}\right)_{e \in \Lambda_{1}}$, indexed over $\Lambda_{1}$ and with $\varphi_{e} \in H_{T \times T}^{*}(G[e] G)$, subject to the additional conditions:

(1) If $f \in E_{2}$ and there is a (necessarily unique) reflection $s_{\alpha_{f}}$ satisfying $s_{\alpha_{f}} f=$ $f s_{\alpha_{f}} \neq f$, then

$$
\varphi_{e_{f}}\left(f_{1} u\right) \equiv \varphi_{e_{f}}\left(f_{2} u\right) \bmod \left(\alpha_{f},-\alpha_{f} \circ \operatorname{int}(u)\right)
$$

for all $u \in W$. Here, $f_{1}$ and $f_{2}=s_{\alpha_{f}} \cdot f_{1} \cdot s_{\alpha_{f}}$ are the two idempotents in $E_{1}$ below $f$, the root $\alpha_{f}$ corresponds to the reflection $s_{\alpha_{f}}$, and $e_{f} \in \Lambda_{1}$ is the unique element of $\Lambda_{1}$ which is conjugate to $f_{1}$.

(2) If $f \in E_{2}$ and $s f=f s=f$ for every $s \in C_{W}(f)$, then

$$
\varphi_{e_{1}}\left(f_{1} u\right) \equiv \varphi_{e_{2}}\left(f_{2} u\right) \bmod \left(\lambda_{f},-\lambda_{f} \circ \operatorname{int}(u)\right)
$$

for all $u \in W$. Here, $\lambda_{f}$ is the character of $T$ defined by the composition

$$
T \rightarrow T f \rightarrow T f / \mathbb{G}_{m} \simeq \mathbb{G}_{m},
$$

the idempotents $f_{1}, f_{2}$ are the unique idempotents below $f$, and $e_{i} \in \Lambda_{1}$ is conjugate to $f_{i}$, for $i=1,2$.

Proof. Since $X$ is a GKM variety (Theorem 3.7) and $X^{T \times T} \subset \bigsqcup_{e \in \Lambda_{1}} G[e] G$, one easily checks that the natural map $H_{T \times T}^{*}(X) \longrightarrow H_{T \times T}^{*}\left(\bigsqcup_{e \in \Lambda_{1}} G[e] G\right)$ is injective. Now we apply Theorem 3.1 to describe the image. First, observe that the curves of type (1) and (2) in Theorem 2.5 are contained in $\bigsqcup_{e \in \Lambda_{1}} G[e] G$, and these curves describe $H_{T \times T}^{*}\left(\bigsqcup_{e \in \Lambda_{1}} G[e] G\right)$ (see e.g. [Br1, Proposition 6.5]). Thus, to conclude the proof, we just need to show that the curves of type (3) in Theorem 2.5 yield assertions (a) and (b). So let $\overline{[T x T]}$, with $x=f u \in \mathcal{R}_{2}$, be one of these curves. By Lemma2.11 either there exists a unique reflection $s_{\alpha_{f}}$ such that $s_{\alpha_{f}} f=f s_{\alpha_{f}} \neq f$, or $s f=f s=f$ for all $s \in C_{W}(f)$. In the first case, Lemma 2.12 implies that the two fixed points of $\overline{[T x T]}$, namely $f_{1} x$ and $f_{2} x$, lie in the same closed $G \times G$-orbit (here recall that $f_{1}, f_{2}$ are the two idempotents below $f$ ). Moreover, $f_{2}$ is conjugate to $f_{1}$ via $s_{\alpha_{f}}$, namely, $f_{2}=s_{\alpha_{f}} \cdot f_{1} \cdot s_{\alpha_{f}}$. We now use Lemma 2.13 to write the associated character $\theta_{x}$ as $\theta_{x}=\left(\alpha_{f},-\alpha_{f} \circ \operatorname{int}(u)\right)$ (in additive notation), where $\alpha_{f}$ is the root associated to the reflection $s_{\alpha_{f}}$. Since $\Lambda_{1}$ indexes all closed $G \times G$-orbits in $X$, there exists a unique $e_{f} \in \Lambda_{1}$ such that $f_{1}$ and $e_{f}$ are conjugate. Assertion (a) is now proved. Finally, if $s f=f s=f$ for all $s \in C_{W}(f)$, then $f_{1}$ and $f_{2}$ are not conjugate (Lemma 2.12). That is, $f_{1} x$ and $f_{2} x$ lie in different closed $G \times G$-orbits. Since $x=f u$, Lemma 2.9 finishes the proof.

The previous result provides a complete combinatorial description of the equivariant cohomology of any rationally smooth projective group embedding. Furthermore, since $X$ is a GKM-variety, the non-equivariant cohomology $H^{*}(X)$ can be recovered from Theorem 3.8 via $H^{*}(X) \simeq H_{T \times T}^{*}(X) \otimes_{S \otimes S} \mathbb{Q}$. As pointed out, Brion [Br3, Theorem 3.1.1] has obtained a result analogous to Theorem 3.8 for projective regular embeddings. Since the latter is a subclass of the class of rationally smooth projective group embeddings, our Theorem 3.8 implies [Br3, Theorem 3.1.1]. 
The $G \times G$-equivariant cohomology of $X$ is obtained by means of the formula $H_{G \times G}^{*}(X) \simeq\left(H_{T \times T}^{*}(X)\right)^{W \times W}$.

Corollary 3.9. If $X=\mathbb{P}_{\epsilon}(M)$ is a rationally smooth group embedding, then the ring $H_{G \times G}^{*}(X)$ consists of all tuples $\left(\psi_{e}\right)_{e \in \Lambda_{1}}$, where $\psi_{e} \in(S \otimes S)^{C_{W}(e) \times C_{W}(e)}$, satisfying the following conditions:

(a) If $f \in \Lambda_{2}$ and there is a (unique) reflection $s_{\alpha_{f}}$ such that $s_{\alpha_{f}} f=f s_{\alpha_{f}} \neq f$, then

$$
\left(s_{\alpha_{f}}, s_{\alpha_{f}}\right) \psi_{e} \equiv \psi_{e} \bmod \left(\alpha_{f},-\alpha_{f}\right)
$$

where $e \leq f$ and the root $\alpha_{f}$ corresponds to the reflection $s_{\alpha_{f}}$.

(b) If $f \in \Lambda_{2}$ and $s f=f s=f$ for every reflection $s \in C_{W}(f)$, then

$$
\psi_{e} \equiv \psi_{e^{\prime}} \bmod \left(\lambda_{f},-\lambda_{f}\right)
$$

where $e, e^{\prime} \leq f$, and $\lambda_{f}$ is the character of $T$ defined by $f$.

Proof. Note that $W \times W$ acts on a tuple $\left(f_{r}\right)$ in $H_{T \times T}^{*}\left(\mathcal{R}_{1}\right)=\bigoplus_{r \in \mathcal{R}_{1}} S \otimes S$ via $(u, v) \cdot\left(f_{r}\right):=\left((u, v) \cdot f_{u^{-1}} r v\right)$. For a subgroup $H$ of $G$, we denote by $E H \rightarrow B H$ the universal principal $H$-bundle. Now let $e \in \Lambda_{1}$. From [Br2, p. 25] it follows that $H^{*}\left(B P_{e}\right) \simeq H^{*}\left(B C_{G}(e)\right) \simeq H^{*}(B T)^{C_{W}(e)}$, because $C_{G}(e)$ is the Levi subgroup of $P_{e}$. Consequently,

$$
\begin{array}{rlc}
H_{G \times G}^{*}(G[e] G) & \simeq & H^{*}\left(B P_{e}\right) \otimes H^{*}\left(B P_{e}^{-}\right) \\
\simeq & H^{*}\left(B C_{G}(e)\right) \otimes H^{*}\left(B C_{G}(e)\right) \\
\simeq & H^{*}(B T)^{C_{W}(e)} \otimes H^{*}(B T)^{C_{W}(e)} \\
\simeq & & (S \otimes S)^{C_{W}(e) \times C_{W}(e)} .
\end{array}
$$

Moreover, one checks that this isomorphism is induced by restriction to the fixed point $[e]$ of $G[e] G$ (cf. [Br1, Section 6.6]). By $W \times W$-invariance, the restriction of $\varphi_{e} \in H_{G \times G}^{*}(G[e] G)$ to the fixed point $(u, v) \cdot e=u^{-1} e v$ is equal to $(u, v) \cdot \psi_{e}$, where $\psi_{e}=\varphi_{e}(e)$. Thus the relations (1) and (2) of Theorem 3.8 reduce to the proposed descriptions (a) and (b).

Let $Y=\mathbb{P}_{\epsilon}(\bar{T}) \subset X$ be the associated toric variety of $X$ (Subsection 1.2). Our next theorem allows to compare the equivariant cohomologies of $X$ and $Y$. The situation for rationally smooth group embeddings contrasts deeply with the corresponding one for regular embeddings, cf. [Br3, Corollary 3.1.2] and [U], Corollary 2.2.3]. It is worth noting that the idea of comparing $Y$ and $X$ goes back to $[\mathrm{LP}]$.

Theorem 3.10. If $X=\mathbb{P}_{\epsilon}(M)$ is rationally smooth, then the inclusion of the associated torus embedding $\iota: Y \hookrightarrow X$ induces an injection:

$$
\iota^{*}: H_{G \times G}^{*}(X) \hookrightarrow H_{T \times T}^{*}(Y)^{W} \simeq\left(H_{T}^{*}(Y) \otimes S\right)^{W},
$$

where the $W$-action on $H_{T \times T}^{*}(Y)$ is induced from the action of $\operatorname{diag}(W)$ on $Y$. Furthermore, $\iota^{*}$ is an isomorphism if and only if $C_{W}(e)=\{1\}$ for every $e \in \Lambda_{1}$. 
Proof. Since $X$ is rationally smooth, then $Y$ is rationally smooth as well (Theorem 3.3). Therefore, we have the following commutative diagram

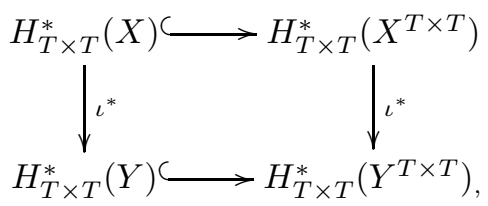

where the horizontal maps are injective, because both group embeddings are GKM. On the other hand, recall that $\Lambda_{1}$ provides a set of representatives of both the $W \times W$-orbits in $X^{T \times T}=\mathcal{R}_{1}$ and the $W$-orbits in $Y^{T \times T}=E_{1}$. Thus, after taking invariants, we obtain an injection

$$
H_{T \times T}^{*}\left(\mathcal{R}_{1}\right)^{W \times W}=\bigoplus_{e \in \Lambda_{1}}(S \otimes S)^{C_{W}(e) \times C_{W}(e)} \hookrightarrow H_{T \times T}^{*}\left(E_{1}\right)^{W}=\bigoplus_{e \in \Lambda_{1}}(S \otimes S)^{C_{W}(e)} .
$$

Placing this information into the commutative diagram above shows that the restriction map

$$
\iota^{*}: H_{T \times T}^{*}(X)^{W \times W} \longrightarrow H_{T \times T}^{*}(Y)^{W}
$$

is injective. Now observe that $H_{T \times T}^{*}(Y)^{W} \simeq\left(H_{T}^{*}(Y) \otimes S\right)^{W}$. Indeed, we have a split exact sequence

$$
1 \longrightarrow \operatorname{diag}(T) \longrightarrow T \times T \frac{\left(t_{1}, t_{2}\right) \mapsto t_{1} t_{2}^{-1}}{\ulcorner-\ldots T} \longrightarrow \text { — }
$$

where the splitting is given by $t \mapsto(t, 1)$. It follows that $T \times T$ is canonically isomorphic to $\operatorname{diag}(T) \times(T \times 1)$. Furthermore, by definition, $\operatorname{diag}(T)$ acts trivially on $Y$. As a consequence, we have a ring isomorphism $H_{T \times T}^{*}(Y) \simeq S \otimes H_{T}^{*}(Y)$. This isomorphism is further $W$-invariant since the $W$-action on the cohomology rings is induced from the action of $\operatorname{diag}(W)$ on $Y$.

To prove the second part of the Theorem, we adapt to our situation an argument of Littelmann and Procesi [LP, Theorem 2.3].

First, assuming that $\iota^{*}$ is also surjective, we need to show that $C_{W}(e)=\{1\}$ for all $e \in \Lambda_{1}$. Since $X$ is equivariantly formal, then $H_{G \times G}^{*}(X)$ is a free $(S \otimes S)^{W \times W}$ module of rank $\left|\mathcal{R}_{1}\right|$ (Lemma 3.2). And $H_{T \times T}^{*}(Y)$ is a free $S \otimes S$-module of rank $\left|E_{1}\right|$, for the same reason. Recall that we can choose a graded $W \times W$-submodule $R$ of $S \otimes S$, isomorphic to the regular representation of $W \times W$, such that

$$
S \otimes S \simeq R \otimes(S \otimes S)^{W \times W}
$$

as graded $(S \otimes S)^{W \times W}$-module. Accordingly, $H_{T \times T}^{*}(Y)^{W}$ is in a natural way a free $(S \otimes S)^{W \times W}$-module. Since, by assumption, $\iota^{*}$ is a graded isomorphism of free $(S \otimes S)^{W \times W}$-modules, we conclude that the ranks of $H_{G \times G}^{*}(X)$ and $H_{T \times T}^{*}(Y)^{W}$ must be the same. The next step consists in finding out a more intrisic formula for the rank of the latter module, so as to compare it with $\left|\mathcal{R}_{1}\right|$.

Since $H_{T \times T}^{*}(Y)$ is a free $S \otimes S$-module, we can find a graded $W$-stable submodule $U$ of $H_{T \times T}^{*}(Y)$ such that the morphism

$$
U \otimes(S \otimes S) \longrightarrow H_{T \times T}^{*}(Y)
$$

is a $W$-equivariant isomorphism of graded $S \otimes S$-modules (cf. proof of Lemma 3.2). Here $\operatorname{dim} U=\operatorname{rank}_{S \otimes S} H_{T \times T}^{*}(Y)=\left|E_{1}\right|$. Hence

$H_{T \times T}^{*}(Y)^{W} \simeq(U \otimes S \otimes S)^{W} \simeq\left(U \otimes R \otimes(S \otimes S)^{W \times W}\right)^{W} \simeq(U \otimes R)^{W} \otimes(S \otimes S)^{W \times W}$. 
Since $R$ decomposes into the direct sum of $|W|$-copies of the regular representation of $W$, then Lemma 3.11 shows that $\operatorname{dim}(U \otimes R)^{W}=\left|E_{1}\right||W|$. Consequently, $H_{T \times T}^{*}(Y)^{W}$ as a free $(S \otimes S)^{W \times W}$-module of rank $\left|E_{1}\right||W|$. In summary, the surjectivity of $\iota^{*}$ implies that $\left|\mathcal{R}_{1}\right|=\left|E_{1}\right||W|$. Now Lemma 3.12 finally yields $C_{W}(e)=\{1\}$, for all $e \in \Lambda_{1}$.

For the converse, suppose that $C_{W}(e)=\{1\}$ for all $e \in \Lambda_{1}$. We need to show that $\iota^{*}$ is surjective. To achieve our goal, we modify slightly an argument of [LP, Section 4.1] and [Br3, Corollary 3.1.2]. Define the variety $\mathcal{N}=\bigcup_{w \in W} w Y$. We claim that this union is, in fact, a disjoint union. Indeed, observe that $\mathcal{N}$ contains all the $T \times T$-fixed points of $X$. That is, $\mathcal{N}$ has $\left|\mathcal{R}_{1}\right|$ fixed points. On the other hand, each $w Y$ has $\left|E_{1}\right|$ fixed points (for its corresponding $T$-action). Now, if it were the case that there is a pair of distinct subvarieties $w Y$ and $w^{\prime} Y$ with non-empty intersection, then this intersection should also contain $T \times T$-fixed points. But then a simple counting argument would yield $\left|\mathcal{R}_{1}\right|<\left|E_{1}\right||W|$. This is impossible, by our assumptions and Lemma 3.12, Hence, $\mathcal{N}=\bigsqcup_{w \in W} w Y$.

Clearly, $\mathcal{N}$ is rationally smooth and equivariantly formal (because each $w Y$ is so, for $w \in W)$. Since $\mathcal{N}$ contains all the $T \times T$-fixed points of $X$, the restriction map

$$
H_{T \times T}^{*}(X) \rightarrow H_{T \times T}^{*}(\mathcal{N})
$$

is injective. Moreover, by Theorem 2.7. $\mathcal{N}$ contains all $T \times T$-curves of $X$ which are not in a closed $G \times G$-orbit, i.e. those which contribute to relations (1) and (2) of Theorem 3.8. As a consequence, restriction to $\mathcal{N}$ induces isomorphisms

$$
H_{T \times T}^{*}(X)^{W \times W} \simeq H_{T \times T}^{*}(\mathcal{N})^{W \times W} \simeq\left(\bigoplus_{w \in W} H_{T \times T}^{*}(Y)\right)^{W \times W} \simeq H_{T \times T}^{*}(Y)^{W} .
$$

The proof is now complete.

Lemma 3.11 ([LP). If $N$ is a finite group, and $U$ and $V$ are two finite dimensional representations of $N$ such that $V$ is the sum of copies of the regular representation of $N$, then

$$
\operatorname{dim}(V \otimes U)^{N}=\frac{\operatorname{dim} V \cdot \operatorname{dim} U}{|N|} .
$$

Lemma 3.12. Let $\mathcal{R}_{1}$ be the set of rank one elements of the Renner monoid $\mathcal{R}$. Then $\left|\mathcal{R}_{1}\right|=\left|E_{1}\right| \cdot|W|$ if and only if $C_{W}(e)=1$ for every $e \in \Lambda_{1}$.

Proof. Recall that $\Lambda_{1}$ can be identified with a set of representatives of the $W \times W$ orbits in $\mathcal{R}_{1}$. Likewise, $\Lambda_{1}$ also corresponds to a set of representatives of the $W$ orbits in $E_{1}$. Let $k$ be the cardinality of $\Lambda_{1}$ and let $e_{1}, \ldots, e_{k}$ be a complete list of the elements of $\Lambda_{1}$. Since we are dealing with elements of rank one, we have $W e_{i} W \simeq\left(W / C_{W}\left(e_{i}\right)\right) \times\left(W / C_{W}\left(e_{i}\right)\right)$, for all $i=1, \ldots, k$. Indeed, consider the maps $\zeta_{i}: W e_{i} W \rightarrow\left(W / C_{W}\left(e_{i}\right)\right) \times\left(W / C_{W}\left(e_{i}\right)\right)$ given by $\zeta_{i}\left(\sigma e_{i} \tau^{-1}\right)=\left(\sigma e_{i} \sigma^{-1}, \tau e_{i} \tau^{-1}\right)$. By the uniqueness of the decomposition in $\mathcal{R}$, the various $\zeta_{i}$ are well-defined and surjective. To check that they are also injective, note that $e_{i} \sigma=e_{i} \sigma=e_{i}$ for any $\sigma \in C_{W}\left(e_{i}\right)$, because $e_{i}$ is minimal. Thus

$$
\left|\mathcal{R}_{1}\right|=\sum_{i}\left|W e_{i} W\right|=\sum_{i}\left|W / C_{W}\left(e_{i}\right)\right|^{2} .
$$


On the other hand, the orbit $W e_{i} \subset E_{1}$ satisfies $W e_{i} \simeq W / C_{W}\left(e_{i}\right)$. This implies the following formula

$$
\left|E_{1}\right|=\sum_{i}\left|W e_{i}\right|=\sum_{i}\left|W / C_{W}\left(e_{i}\right)\right| .
$$

The result now follows.

Next we characterize those group embeddings for which the map $\iota^{*}$ of Theorem 3.10 is an isomorphism.

Proposition 3.13. Let $X=\mathbb{P}_{\epsilon}(M)$ be a projective group embedding. Let $Y=$ $\mathbb{P}_{\epsilon}(\bar{T})$ be the associated torus embedding and $\iota: Y \rightarrow X$ the canonical inclusion. Then following are equivalent:

(a) $C_{W}(e)=\{1\}$ for every $e \in E_{1}$.

(b) All closed $G \times G$-orbits in $X$ are isomorphic to $G / B \times G / B^{-}$.

If, moreover, $X$ is rationally smooth, then (a) and (b) are equivalent to

(c) The induced map $\iota^{*}: H_{G \times G}^{*}(X) \rightarrow H_{T \times T}^{*}(Y)^{W} \simeq\left(H_{T}^{*}(Y) \otimes S\right)^{W}$ is an isomorphism.

Proof. For the equivalence between (a) and (b) remember that every closed $G \times G$ orbit in $X$ is of the form $G / P_{e} \times G / P_{e}^{-}$, for $e \in E_{1}$. Also, recall that $C_{G}(e)$, the common Levi subgroup of $P_{e}$ and $P_{e}^{-}$, has Weyl group equal to $C_{W}(e)$. Then $C_{W}(e)=\{1\}$, for all $e \in E_{1}$, if and only if $P_{e}$ and $P_{e}^{-}$are two mutually opposite Borel subgroups containing $T$, for all $e \in E_{1}$. If, in addition, $X$ is rationally smooth, then the equivalence between statements (c) and (a) follows at once from Theorem [3.10, since $\Lambda_{1}$ is the set of representatives of the $W$-orbits in $E_{1}$.

Group embeddings satisfying the equivalent conditions (a) and (b) of Proposition 3.13 are called toroidal group embeddings, see [T2, Section 29]. Observe that smooth toroidal group embeddings are exactly the regular embeddings of reductive groups [T2, Theorem 29.2]. Our Theorem 3.10 states that the results of [LP, [U] and [Br3] can be extended to the class of toroidal rationally smooth projective group embeddings. Furthermore, Theorem 3.10 gives a precise relation between our results and those on simplicial toric varieties. Indeed, if $X=\mathbb{P}_{\epsilon}(M)$ is a toroidal rationally smooth group embedding, then $H_{G \times G}^{*}(X)$ is isomorphic to the subring of $W$-invariants in $H_{T}^{*}(Y) \otimes S$, where $H_{T}^{*}(Y)$ is the ring of piecewise polynomial functions on the fan of $Y=\mathbb{P}_{\epsilon}(\bar{T})$, see $[\mathrm{BV}]$.

We conclude this section describing the non-equivariant cohomology ring of toroidal rationally smooth group embeddings. This result is known for regular embeddings, by work of De Concini-Procesi [DP] and Littelmann-Procesi [LP].

Theorem 3.14. Let $M$ be a reductive monoid with zero and unit group $G$. Let $K$ be a maximal compact subgroup of $G$ such that $T_{K}=T \cap K$ is a maximal compact torus. Suppose that the projective group embedding $X=\mathbb{P}_{\epsilon}(M)$ is toroidal and rationally smooth. Then

$$
H^{*}(X) \simeq H^{*}\left((K \times K) \times_{\left(T_{K} \times T_{K}\right)} Y\right)^{W},
$$

where $Y=\mathbb{P}_{\epsilon}(\bar{T}) \subset X$ is the associated toric variety. 
Proof. As $G / K$ is contractible, the functors $H_{G \times G}^{*}(-)$ and $H_{K \times K}^{*}(-)$ agree on $G \times G$-spaces. Similar remarks apply to $H_{T \times T}^{*}(-)$ and $H_{T_{K} \times T_{K}}^{*}(-)$, for $T / T_{K}$ is also contractible. Since $X$ is toroidal and rationally smooth, Theorem 3.10 yields

$$
H^{*}(X) \simeq H_{T_{K} \times T_{K}}^{*}(Y)^{W} / \mathcal{I} H_{T_{K} \times T_{K}}^{*}(Y)^{W},
$$

where $\mathcal{I}$ is the ideal of $(S \otimes S)^{W \times W}$ generated by the elements of strictly positive degree. As pointed out in [LP, Remark 2.3], the induction formula Q, p. 552] implies that

$$
H_{T_{K} \times T_{K}}^{*}(Y)=H_{K \times K}^{*}\left((K \times K) \times_{T_{K} \times T_{K}} Y\right) .
$$

and the latter is isomorphic to

$$
(S \otimes S)^{W \times W} \otimes_{\mathbb{Q}} H^{*}\left((K \times K) \times_{T_{K} \times T_{K}} Y\right),
$$

because $(K \times K) \times_{T_{K} \times T_{K}} Y$ has no cohomology in odd degrees. Hence,

$$
H_{T_{K} \times T_{K}}^{*}(Y)^{W} \simeq(S \otimes S)^{W \times W} \otimes_{\mathbb{Q}} H^{*}\left((K \times K) \times_{T_{K} \times T_{K}} Y\right)^{W},
$$

and thus

$$
H_{T_{K} \times T_{K}}^{*}(Y)^{W} / \mathcal{I} H_{T_{K} \times T_{K}}^{*}(Y)^{W} \simeq H^{*}\left((K \times K) \times_{T_{K} \times T_{K}} Y\right)^{W} .
$$

We conclude that $H^{*}(X) \simeq H^{*}\left((K \times K) \times_{T_{K} \times T_{K}} Y\right)^{W}$.

\section{Simple GROUP EMBEDDingS}

Let $H$ be a connected reductive group. Recall that an embedding of $H$ is called simple if it contains only one closed $H \times H$-orbit.

4.1. Rationally smooth simple group embeddings. Classification. A reductive monoid $M$ with 0 is called J-irreducible if $M \backslash\{0\}$ has exactly one minimal $G \times G$-orbit. Equivalently, there is a unique minimal nonzero idempotent $e_{1} \in E(\bar{T})$ such that $\Lambda_{1}=\left\{e_{1}\right\}$. It follows that $E_{1} \simeq W / C_{W}\left(e_{1}\right)$, and $f e_{1}=e_{1}$ for all $f \in \Lambda \backslash\{0\}$ (Subsection 1.1). Clearly, simple projective group embeddings are exactly the projectivizations of J-irreducible monoids (Subsection 1.2). See [PR] or [R8, Section 7.3] for a systematic discussion of J-irreducible monoids. Due to the following result, any $\mathcal{J}$-irreducible monoid is semisimple and can be obtained via the procedure of Example 1.1

Theorem 4.1 ([PR $)$. A reductive monoid $M$, with zero, is J-irreducible if and only if there is an irreducible rational representation $\rho: M \rightarrow E n d(V)$ which is finite as a morphism of algebraic varieties.

Let $M$ be a $\mathcal{J}$-irreducible monoid with $\Lambda_{1}=\left\{e_{1}\right\}$, as above. We say that $M$ is J-irreducible of type $J$ if $J=\left\{s \in \Sigma \mid s e_{1}=e_{1} s\right\}$, where $\Sigma$ is the set of simple reflections of $W$. Notice that $C_{W}\left(e_{1}\right)=W_{J}$, the subgroup of $W$ generated by $J$. The set $J$ can be determined in terms of any irreducible representation satisfying Theorem 4.1. It is worthwhile to pause and notice that $\Lambda$ is completely determined by $J$.

Theorem $4.2([\mathrm{PR}])$. If $M$ is a J-irreducible monoid of type $J$, then

$\Lambda \backslash\{0\} \simeq\{I \subseteq \Sigma \mid$ no connected component of $I$ is contained entirely in $J\}$,

in such a way that e corresponds to $I \subseteq \Sigma$ if $I=\{s \in \Sigma \mid$ se $=e s \neq e\}$. This bijection identifies $\Lambda_{2}$ with $\Sigma \backslash J$. 
Let $X=\mathbb{P}(M)$ be a simple projective embedding, where $M$ is a $\mathcal{J}_{\text {-irreducible }}$ monoid, $\Lambda_{1}=\left\{e_{1}\right\}$, and $J=\left\{s \in \Sigma \mid s e_{1}=e_{1} s\right\}$. Then $P_{e_{1}}=\bigsqcup_{w \in W_{J}} B w B=P_{J}$, where $P_{J} \subset G$ is the standard parabolic subgroup associated to $J$. Hence, by Theorem 1.2, the unique closed orbit of $X$ is $G\left[e_{1}\right] G \simeq G / P_{J} \times G / P_{J}^{-}$. Note that $X$ is toroidal only when $J=\emptyset$ (Proposition 3.13). In general, the $G \times G$-orbit structure of $X=\mathbb{P}(M)$ is completely determined by $J$ (Theorem 4.2).

Definition 4.3. Let $X$ be a simple projective group embedding. We say that $X$ is simple of type $J$ if $X=\mathbb{P}(M)$, where $M$ is a $\mathcal{J}$-irreducible monoid of type $J$.

The type of a simple group embedding is independent of its presentation as a projectivization of a monoid [R7]. Renner [R5, R7] has classified all rationally smooth simple group embeddings according to their type. Below is the list.

Theorem 4.4 ([R7, Proposition 2.8]). For each irreducible Dynkin diagram the following is a list of all types, $J \subset \Sigma$, of J-irreducible monoids $M$ of type $J$ such that $\mathbb{P}(M)$ is rationally smooth. The numbering of the elements of $\Sigma$ is as follows. For types $A_{n}, B_{n}, C_{n}, F_{4}$, and $G_{2}$ it is the usual numbering. In these cases the end nodes are $s_{1}$ and $s_{n}$. For type $E_{6}$ the end nodes are $s_{1}, s_{5}$ and $s_{6}$ with $s_{3} s_{6} \neq s_{6} s_{3}$. For type $E_{7}$ the end nodes are $s_{1}, s_{6}$ and $s_{7}$ with $s_{4} s_{7} \neq s_{7} s_{4}$. For type $E_{8}$ the end nodes are $s_{1}, s_{7}$ and $s_{8}$ with $s_{5} s_{8} \neq s_{8} s_{5}$. In each case of type $E_{n}$, the nodes corresponding to $s_{1}, s_{2}, \ldots, s_{n-1}$ determine the unique subdiagram of type $A_{n-1}$. For type $D_{n}$ the end nodes are $s_{1}, s_{n-1}$ and $s_{n}$. The two subdiagrams of $D_{n}$, of type $A_{n-1}$, correspond to the subsets $\left\{s_{1}, s_{2}, \ldots, s_{n-2}, s_{n-1}\right\}$ and $\left\{s_{1}, s_{2}, \ldots, s_{n-2}, s_{n}\right\}$ of $\Sigma$. For $s_{i} \in \Sigma$, the corresponding simple root is denoted $\alpha_{i}$.

(1) $A_{n}, n \geq 1$. Let $\Sigma=\left\{s_{1}, \ldots, s_{n}\right\}$.

(a) $J=\phi$.

(b) $J=\left\{s_{1}, \ldots, s_{i}\right\}, 1 \leq i<n$.

(c) $J=\left\{s_{j}, \ldots, s_{n}\right\}, 1<j \leq n$.

(d) $J=\left\{s_{1}, \ldots, s_{i}, s_{j}, \ldots s_{n}\right\}, 1 \leq i, i \leq j-3$ and $j \leq n$.

(2) $B_{n}, n \geq 2$. Let $\Sigma=\left\{s_{1}, \ldots, s_{n}\right\}, \alpha_{n}$ short.

(a) $J=\phi$.

(b) $J=\left\{s_{1}, \ldots, s_{i}\right\}, 1 \leq i<n$.

(c) $J=\left\{s_{n}\right\}$.

(d) $J=\left\{s_{1}, \ldots, s_{i}, s_{n}\right\}, 1 \leq i$ and $i \leq n-3$.

(3) $C_{n}, n \geq 3$. Let $\Sigma=\left\{s_{1}, \ldots, s_{n}\right\}$, $\alpha_{n}$ long.

(a) $J=\phi$.

(b) $J=\left\{s_{1}, \ldots, s_{i}\right\}, 1 \leq i<n$.

(c) $J=\left\{s_{n}\right\}$.

(d) $J=\left\{s_{1}, \ldots, s_{i}, s_{n}\right\}, 1 \leq i$ and $i \leq n-3$.

(4) $D_{n}, n \geq 4$. Let $\Sigma=\left\{s_{1}, \ldots s_{n-2}, s_{n-1}, s_{n}\right\}$.

(a) $J=\phi$.

(b) $J=\left\{s_{1}, \ldots, s_{i}\right\}, 1 \leq i \leq n-3$.

(c) $J=\left\{s_{n-1}\right\}$.

(d) $J=\left\{s_{n}\right\}$.

(e) $J=\left\{s_{1}, \ldots, s_{i}, s_{n-1}\right\}, 1 \leq i \leq n-4$.

(f) $J=\left\{s_{1}, \ldots, s_{i}, s_{n}\right\}, 1 \leq i \leq n-4$.

(5) $E_{6}$. Let $\Sigma=\left\{s_{1}, s_{2}, s_{3}, s_{4}, s_{5}, s_{6}\right\}$.

(a) $J=\phi$.

(b) $J=\left\{s_{1}\right\}$ or $\left\{s_{1}, s_{2}\right\}$. 
(c) $J=\left\{s_{5}\right\}$ or $\left\{s_{4}, s_{5}\right\}$.

(d) $J=\left\{s_{6}\right\}$.

(e) $J=\left\{s_{1}, s_{5}\right\},\left\{s_{1}, s_{2}, s_{5}\right\}$ or $\left\{s_{1}, s_{4}, s_{5}\right\}$.

(f) $J=\left\{s_{1}, s_{6}\right\}$.

(g) $J=\left\{s_{5}, s_{6}\right\}$

(h) $J=\left\{s_{1}, s_{5}, s_{6}\right\}$.

(6) $E_{7}$. Let $\Sigma=\left\{s_{1}, s_{2}, s_{3}, s_{4}, s_{5}, s_{6}, s_{7}\right\}$.

(a) $J=\phi$.

(b) $J=\left\{s_{1}\right\},\left\{s_{1}, s_{2}\right\}$ or $\left\{s_{1}, s_{2}, s_{3}\right\}$.

(c) $J=\left\{s_{6}\right\}$ or $\left\{s_{5}, s_{6}\right\}$.

(d) $J=\left\{s_{7}\right\}$.

(e) $J=\left\{s_{1}, s_{6}\right\},\left\{s_{1}, s_{2}, s_{6}\right\},\left\{s_{1}, s_{2}, s_{3}, s_{6}\right\},\left\{s_{1}, s_{5}, s_{6}\right\}$, or $\left\{s_{1}, s_{2}, s_{5}, s_{6}\right\}$.

(f) $J=\left\{s_{6}, s_{7}\right\}$.

(g) $J=\left\{s_{1}, s_{7}\right\}$ or $\left\{s_{1}, s_{2}, s_{7}\right\}$.

(h) $J=\left\{s_{1}, s_{6}, s_{7}\right\},\left\{s_{1}, s_{2}, s_{6}, s_{7}\right\}$.

(7) $E_{8}$. Let $\Sigma=\left\{s_{1}, s_{2}, s_{3}, s_{4}, s_{5}, s_{6}, s_{7}, s_{8}\right\}$.

(a) $J=\phi$.

(b) $J=\left\{s_{1}\right\},\left\{s_{1}, s_{2}\right\},\left\{s_{1}, s_{2}, s_{3}\right\}$ or $\left\{s_{1}, s_{2}, s_{3}, s_{4}\right\}$.

(c) $J=\left\{s_{7}\right\}$ or $\left\{s_{6}, s_{7}\right\}$.

(d) $J=\left\{s_{8}\right\}$.

(e) $J=\left\{s_{1}, s_{7}\right\},\left\{s_{1}, s_{2}, s_{7}\right\},\left\{s_{1}, s_{2}, s_{3}, s_{7}\right\},\left\{s_{1}, s_{2}, s_{3}, s_{4}, s_{7}\right\}$, $\left\{s_{1}, s_{6}, s_{7}\right\},\left\{s_{1}, s_{2}, s_{6}, s_{7}\right\},\left\{s_{1}, s_{2}, s_{3}, s_{6}, s_{7}\right\}$ or $\left\{s_{1}, s_{2}, s_{5}, s_{6}\right\}$.

(f) $J=\left\{s_{7}, s_{8}\right\}$.

(g) $J=\left\{s_{1}, s_{8}\right\},\left\{s_{1}, s_{2}, s_{8}\right\}$ or $\left\{s_{1}, s_{2}, s_{3}, s_{8}\right\}$.

(h) $J=\left\{s_{1}, s_{7}, s_{8}\right\},\left\{s_{1}, s_{2}, s_{7}, s_{8}\right\}$.

(8) $F_{4}$. Let $\Sigma=\left\{s_{1}, s_{2}, s_{3}, s_{4}\right\}$.

(a) $J=\phi$.

(b) $J=\left\{s_{1}\right\}$ or $\left\{s_{1}, s_{2}\right\}$.

(c) $J=\left\{s_{4}\right\}$ or $\left\{s_{3}, s_{4}\right\}$.

(d) $J=\left\{s_{1}, s_{4}\right\}$.

(9) $G_{2}$. Let $\Sigma=\left\{s_{1}, s_{2}\right\}$.

(a) $J=\phi$.

(b) $J=\left\{s_{1}\right\}$.

(c) $J=\left\{s_{2}\right\}$.

According to this list, if $G$ is a semisimple group of adjoint type, then the choice $J=\emptyset$ yields the wonderful compactification of $G$. On the other hand, in type $A_{n}$, the choice $J=\left\{s_{2}, \ldots, s_{n}\right\}$ yields $\mathbb{P}^{(n+1)^{2}-1}$, a compactification of $P G L_{n+1}$.

Remark 4.5. Projective group embeddings are also described in terms of weight polytopes [T1. It is possible to state Theorem 4.4 in that language as well. Indeed, let $M$ be a J-irreducible monoid. Now let $\rho: M \rightarrow \operatorname{End}(V)$ be an irreducible representation as in Theorem 4.1. Let $G_{0}$ be the semisimple part of $G$, with maximal torus $T_{0}=G_{0} \cap T$, and let $\rho_{\lambda}=\rho \mid G_{0}$ with highest weight $\lambda \in \mathcal{C}$, the rational Weyl chamber of $G_{0}$. One checks that $J=\{s \in \Sigma \mid s(\lambda)=\lambda\}$ (see e.g. [R5]). So we can identify $e_{1}$ with $\lambda$. Now the weight polytope $\mathcal{P}_{\lambda}$ is defined to be the convex hull of $W \cdot \lambda$ in $X\left(T_{0}\right) \otimes \mathbb{Q}$, where $X\left(T_{0}\right)$ is the character group of $T_{0}$. This $W$-invariant polytope is considered in T1. Observe that $\mathcal{P}_{\lambda}$ is the 
polytope associated to the toric variety $\mathbb{P}(\bar{T})$. In particular, $E_{1} \simeq W / C_{W}\left(e_{1}\right)$ corresponds to the set of vertices of $\mathcal{P}_{\lambda}$. But even more is true. By R7, Corollary 2.3] the face lattice $\mathcal{F}_{\lambda}$ of $\mathcal{P}_{\lambda}$ is completely described in terms of the Weyl group $(W, \Sigma)$. Indeed, the set of $W$-orbits of $\mathcal{F}_{\lambda}$ is in one-to-one correspondance with $\{I \subseteq \Sigma \mid$ no connected component of $I$ is contained entirely in $J\}$. The latter is $\Lambda \backslash\{0\}$, by Theorem 4.2. The correspondance assigns to $I \subseteq \Sigma$ the unique face $F \in \mathcal{F}_{\lambda}$ with $I=\{s \in \Sigma \mid s(F)=F$ and $s \mid F \neq i d\}$ whose relative interior $F^{0}$ has nonempty intersection with $\mathcal{C}$. It follows from Theorem 3.3 that $\mathbb{P}(M)$ is rationally smooth if and only if $\mathcal{P}_{\lambda}$ is a simple polytope, i.e. there are exactly $|\Sigma|$ edges of $\mathcal{P}_{\lambda}$ meeting at $\lambda$. Observe that $\Sigma \backslash J$ corresponds to the set of fundamental dominant weights involved in the description of $\rho_{\lambda}$; that is, $\lambda$ is a dominant weight of the form $\lambda=\sum m_{i} \lambda_{i}$, where $\lambda_{i}$ runs over the fundamental weights attached to $s_{i} \in \Sigma \backslash J$, and $m_{i}$ are positive (nonzero) numbers. For group embeddings with more than one closed orbit there is certainly a dictionary between Timashev's description and Renner's. The interested reader should consult [R8, Section 7.2], [R7] and [R5]. We shall not pursue this here.

4.2. Equivariant cohomology of simple group embeddings. Let $X=\mathbb{P}(M)$ be a simple embedding of type $J$. Given that $X$ has only one closed orbit, we can associate to any $f \in E_{2}$ a unique reflection $s_{\alpha_{f}}$ such that $s_{\alpha_{f}} f=f s_{\alpha_{f}} \neq f$ (Lemma 2.11 and 2.12). Let $\Lambda_{1}=\left\{e_{1}\right\}$ and put

$$
L^{J}=\left\{f \in E_{2} \mid f e_{1}=e_{1}\right\} .
$$

Theorem 4.6. Let $X=\mathbb{P}(M)$ be a simple embedding of type J. If $X$ is rationally smooth, then the natural morphism

$$
H_{T \times T}^{*}(X) \rightarrow H_{T \times T}^{*}\left(G / P_{J} \times G / P_{J}^{-}\right)
$$

is injective, and its image consists of all maps $\varphi \in H_{T \times T}^{*}\left(G / P_{J} \times G / P_{J}^{-}\right)$, subject to the condition: for every $f \in L^{J}, u \in W$, and $v \in W$, the following holds

$$
\varphi\left(u e_{1} v\right) \equiv \varphi\left(u s_{\alpha_{f}} e_{1} s_{\alpha_{f}} v\right) \bmod \left(\alpha_{f} \circ \operatorname{int}\left(u^{-1}\right),-\alpha_{f} \circ \operatorname{int}(v)\right),
$$

where $\alpha_{f}$ is the root associated to the reflection $s_{\alpha_{f}}$.

Proof. The first assertion is a direct consequence of Theorem 3.8. Besides, $X$ contains a unique closed $G \times G$-orbit, so to describe the image we just need to focus on translating condition (1) of Theorem 3.8 into our situation. Let $f \in E_{2}$. Then there are exactly two rank-one idempotents $f_{1}, f_{2}$, such that $f_{1} f=f_{1}, f_{2} f=f_{2}$ and $f_{2}=s_{\alpha} f_{1} s_{\alpha}$, where $s_{\alpha} f=s_{\alpha} f \neq f$. On the other hand, because $\Lambda_{1}=\left\{e_{1}\right\}$, then $f_{1}=u e_{1} u^{-1}$, for some $u \in W$. The latter implies that $f^{\prime}=u^{-1} f u$ is an idempotent of $\bar{T}$ such that $f^{\prime} e_{1}=e_{1}$, that is, $f^{\prime} \in L^{J}$. In short, any $f \in E_{2}$ is conjugate to an element of $L^{J}$. This observation and Theorem 3.8 (1) yield the result.

Corollary 4.7. Let $X=\mathbb{P}(M)$ be a rationally smooth simple embedding of type $J$. Let $e_{1}$ be the unique rank-one idempotent for which $\Lambda_{1}=\left\{e_{1}\right\}$. Then the ring $H_{G \times G}^{*}(X)$ consists of all $\varphi \in(S \otimes S)^{W_{J} \times W_{J}}$ such that

$$
\left(s_{\alpha_{f}}, s_{\alpha_{f}}\right) \varphi \equiv \varphi \bmod \left(\alpha_{f},-\alpha_{f}\right),
$$

for every $f \in \Lambda_{2} \subset L^{J}$.

Proof. Simply translate Corollary 3.9 into this situation, using Theorem 4.6 , 


\section{REFERENCES}

[BK] Brion, M., Kumar, S. Frobenius splitting methods in geometry and representation theory. Progress in Mathematics, 231. Birkhäuser Boston.

[Bo] Borel, A. Seminar on transformation groups. Annals of Math Studies, No. 46, Princeton University Press, Princeton, N.J. 1960.

[Br1] Brion, M. Equivariant Chow groups for torus actions. Transform. Groups, vol. 2, No. 3, 1997, pp. 225-267.

[Br2] Brion, M. Equivariant cohomology and equivariant intersection theory. Notes by Alvaro Rittatore. NATO Adv. Sci. Inst. Ser. C Math. Phys. Sci., 514, Representation theories and algebraic geometry (Montreal, PQ, 1997), 1-37, Kluwer Acad. Publ., Dordrecht, 1998.

[Br3] Brion, M. The behaviour at infinity of the Bruhat decomposition. Comment. Math. Helv. 73, 1998, pp. 137-174.

[Br4] Brion, M. Rational smoothness and fixed points of torus actions. Transform. Groups, Vol. 4, No. 2-3, 1999, pp. 127-156.

[Br5] Brion, M. Poincaré duality and equivariant cohomology. Michigan Math. J. 48, 2000, pp. 77-92.

[Br6] Brion, M. Local structure of algebraic monoids. Mosc. Math. J. 8 (2008), no. 4, 647-666, 846.

[Br7] Brion, M. On algebraic semigroups and monoids. In Algebraic Monoids, Group Embeddings and Algebraic Combinatorics, vol. 71 of Fields Institute Communication Series, pp. 1-55. Springer, 2014.

[BV] Brion, M., Vergne, M. An equivariant Riemann-Roch theorem for complete, simplicial toric varieties. J. Reine Angew. Math. 482 (1997), 6792.

[C] Carrell, J. The Bruhat graph of a Coxeter group, a conjecture of Deodhar, and rational smoothness of Schubert varieties. Algebraic groups and their generalizations: classical methods (University Park, PA, 1991), 5361, Proc. Sympos. Pure Math., 56, Part 1, Amer. Math. Soc., Providence, RI, 1994.

[CK] Carrell, J.; Kuttler, J. Smooth points of $T$-stable varieties in $G / B$ and the Peterson map. Invent. Math. 151 (2003), no. 2, 353379.

[D] Danilov, V. The geometry of toric varieties. Uspekhi Mat. Nauk 33 (1978), no. 2(200), $85134,247$.

[DP] De Concini,C., Procesi, C. Cohomology of compactifications of algebraic groups. Duke Math. J. 53 (1986), no. 3, 585-594.

[G1] Gonzales, R. Rational smoothness, cellular decompositions and GKM theory. Geometry and Topology, Vol. 18, No. 1 (2014), 291-326.

[G2] Gonzales, R. Localization in equivariant operational K-theory and the Chang-Skjelbred property. Max-Planck-Institut für Mathematik Preprint, 2014-20. arXiv:1403.4412.

[G3] Gonzales, R. Algebraic rational cells, equivariant intersection theory, and Poincaré duality. Max-Planck-Institut für Mathematik Preprint, 2014-21. arXiv:1404.2486.

[GKM] Goresky, M., Kottwitz, R., MacPherson, R. Equivariant cohomology, Koszul duality, and the localization theorem. Invent. math. 131, 25-83 (1998)

[Hs] Hsiang, Wu Yi. Cohomology Theory of Topological Transformation Groups. Ergebnisse der Mathematik und ihrer Grenzgebiete, Band 85. Springer-Verlag, New YorkHeidelberg, 1975.

[Hu] Humphreys, J. Reflection groups and Coxeter groups. Cambridge Studies in Advanced Mathematics, 29. Cambridge University Press, Cambridge, 1990.

[LP] Littelmann, P.; Procesi, C. Equivariant Cohomology of Wonderful Compactifications. Operator algebras, unitary representations, enveloping algebras, and invariant theory (Paris, 1989), 219262, Progr. Math., 92, Birkhäuser Boston, Boston, MA, 1990.

[P1] Perrin, N. On the geometry of spherical varieties. Transform. Groups 19 (2014), no. 1, 171-223.

[P2] Perrin, N. Split subvarieties of group embeddings. Trans. Amer. Math. Soc. (2015).

[PR] M. S. Putcha and L. E. Renner, The system of idempotents and lattice of J-classes of reductive algebraic monoids, Journal of Algebra 116 (1988), 385-399.

[Pu] M. S. Putcha, "Linear Algebraic Monoids", Cambridge University Press, 1988. 
[Q] D. Quillen. The Spectrum of an Equivariant Cohomology Ring: I. The Annals of Mathematics, 2nd. Ser., Vol. 94, No. 3. Nov., 1971, pp. 549-572.

[R1] Renner, L. Classification of semisimple algebraic monoids. Trans. Amer. Math. Soc. 292 (1985), 193-223.

[R2] Renner, L. Analogue of the Bruhat decomposition for algebraic monoids, Journal of Algebra 101(1986), 303-338.

[R3] Renner, L. Classification of semisimple varieties. J. of Algebra, vol. 122, No. 2, 1989, 275-287.

[R4] Renner, L. The H-polynomial of a semisimple monoid. J. Alg. 319 (2008), 360-376.

[R5] Renner, L. Descent systems for Bruhat posets. J. of Alg. Comb., 29 (2009), 413-435.

[R6] Renner, L. Rationally smooth algebraic monoids. Semigroup Forum 78 (2009), 384-395.

[R7] Renner, L. The H-polynomial of an Irreducible Representation. Journal of Algebra 332 (2011) 159-186.

[R8] Renner, L. Linear Algebraic Monoids. Encyclopaedia of Mathematical Sciences, 134. Invariant Theory and Algebraic Transformation Groups, V. Springer, Berlin, 2005.

[Ri] Rittatore, A. Algebraic monoids and group embeddings. Transformation Groups, Vol. 3, No. 4, 1998, pp. 375-396.

[So] Solomon, L. An introduction to reductive monoids. Semigroups, Formal languages and Groups, 295-352, 1995.

[Sp] Springer, T. Linear Algebraic Groups. Birkhäuser Classics. 2nd Edition.

[T1] Timashev, D. Equivariant compactifications of reductive groups. Mat. Sb. 194 (2003), no. 4, 119-146; translation in Sb. Math. 194 (2003), no. 3-4, 589-616.

[T2] Timashev, D. Homogeneous spaces and equivariant embeddings. Encyclopaedia of Mathematical Sciences, 138. Invariant Theory and Algebraic Transformation Groups, 8. Springer, Heidelberg, 2011.

[U] Uma, V. Equivariant K-theory of compactifications of algebraic groups. Transform. Groups 12 (2007), No. 2, 371-406.

Mathematisches Institut, Heinrich-Heine-Universität, 40225 Düsseldorf, Germany

E-mail address: rgonzalesv@gmail.com 